\title{
Towards Essential Hand Tremor Suppression via Pneumatic Artificial Muscles ${ }^{\dagger}$
}

\author{
Vasileios Skaramagkas $^{1}\left[\right.$, George Andrikopoulos ${ }^{2, *} \mathbb{D}$ and Stamatis Manesis ${ }^{3}$ \\ 1 Institute of Computer Science, Foundation of Research \& Technology Hellas (FORTH), 70013 Heraklion, \\ Greece; vskaramag@ics.forth.gr \\ 2 Machine Design Department, KTH Royal Institute of Technology, 11428 Stockholm, Sweden \\ 3 Electrical and Computer Engineering Department, University of Patras, 26500 Patras, Greece; \\ stam.manesis@ece.upatras.gr \\ * Correspondence: geoand@kth.se; Tel.: +46-87906331 \\ + This paper is an extended version of our paper published in European Control Conference (ECC 2020).
}

Citation: Skaramagkas, V.;

Andrikopoulos, G.; Manesis, S.

Towards Essential Hand Tremor Suppression via Pneumatic Artificial Muscles. Actuators 2021, 10, 206.

https://doi.org/10.3390/act10090206

Academic Editor: Alexander Hošovský

Received: 8 July 2021

Accepted: 24 August 2021

Published: 26 August 2021

Publisher's Note: MDPI stays neutral with regard to jurisdictional claims in published maps and institutional affiliations.

Copyright: (c) 2021 by the authors. Licensee MDPI, Basel, Switzerland. This article is an open access article distributed under the terms and conditions of the Creative Commons Attribution (CC BY) license (https:// creativecommons.org/licenses/by/ $4.0 /)$.

\begin{abstract}
Essential tremor (ET) is one of the most common movement disorders and can occur unexpectedly and develop indefinitely to any population unit. According to the recorded statistics of people suffering from ET, the disorder affects $5 \%$ of people worldwide, thus creating an everincreasing need to investigate ways for its suppression and treatment. In this article, we investigate the capability of Pneumatic Artificial Muscles (PAMs) to reduce or even suppress ET leading to the relief of the sufferers. In our work, we designed and constructed two iterations of a glovelike setup and attempted to explore the possibility of suppressing ET on different parts of the hand by exerting force on the index finger and metacarpal region. For both glove iterations, we established an experimental protocol based on the adjustment of a force controller. Finally, we evaluated exhaustively the performance of our setup under multiple motion scenarios with the participation of an ET-diagnosed volunteer.
\end{abstract}

Keywords: essential tremor suppression; pneumatic artificial muscles; soft robotics; orthotic devices

\section{Introduction}

Essential tremor (ET) disorder is a neurological condition that causes the hands to shake rhythmically with a relatively constant frequency and variable amplitude [1,2]. The head, trunk, and voice may also be involved, but hand shaking is most prominent. Based on recorded statistics, ET has often been reported as the most common movement disorder, as almost $5 \%$ of a country's population suffers from it [3]. ET appears most frequently on the upper limbs and as a $4-12 \mathrm{~Hz}$ bilateral postural and kinetic tremor of the hands [4], while no population has been proven to be immune to its development [5]. The cause is not known, but it is often passed down from a parent to a child. Therefore, and as the frequency of ET is rising proportionally with age, the need for its suppression and treatment is vastly growing.

A number of methods have been introduced for the suppression and treatment of ET, as well as other related tremor disorders (e.g., Parkinson's). They mostly include the following: (i) pharmacotherapy, i.e., medication therapy ranging from antiepileptics to antibiotics and bronchodilators; (ii) surgical treatments, with thalamotomy and Deep Brain Stimulation (DBS) reported also in cases of refractory ET [5]. From the existing medication therapies for treating tremor, only a few agents have enough data available for their use to be widely recommended, while surgical interventions may be associated with more severe or persistent side effects.

During the last decade, the evolution of technology in an exponential pace has led to the investigation of alternative ways to suppress tremor. As a result, passive and active orthotic devices have been developed and manufactured, including exoskeletons 
capable of reducing different tremor characteristics (amplitude, frequency) on different joints of the upper limbs: fingers [6-8], wrist $[9,10]$, elbow [11-15], as well as multiple upper-limb joints [16-18]. Moreover, research-oriented and commercial solutions aim at ET suppression during tasks of everyday life such as food-consuming activities [19-21] or handwriting [22]. While all appliances presented potential in reducing tremor effects, their evaluation has only been performed in a controlled lab setting and they have not yet been tested on diagnosed patients. Moreover, some of the devices were characterized by rigid and bulky structures, with constrained applicability to task-specific applications, and limited bandwidth in terms of suppressing higher-frequency tremors. Furthermore, the functionality and ability of devices to avoid user injury under the effect of disturbances during daily use remains a critical matter [23].

In this article, a soft exoskeletal glove for investigating the potential of utilizing Pneumatic Artificial Muscles (PAMs) for suppressing the hand tremor of ET patients is presented. ET has been specifically selected for this investigation, due to its increasing frequency of development in large parts of populations, as well as its relatively higher tremor frequencies and amplitudes [2], which make it a challenging target. Moreover, PAMs have been selected as the actuation method for their similar properties with those of the organic muscle, combined with advantages such as the ability to provide high power outputs with light weights and inherent compliance [24]. Moreover, PAMs meet the need for safety, simplicity, and lightness that human-robot interaction requires, which justifies its utilization in medical and biorobotic applications.

This article's contributions stem from the design and the development of a hand exoskeleton that utilizes soft actuators for force-resistive applications. The exoskeleton's design combines a lightweight approach with high efficiency and modularity, thus offering increased performance in various hand-motion scenarios. In this article, the hand tremor suppression potential by applying kinesthetic force on the user's fingertips and on the metacarpal region is investigated. The presented approach, which extends the findings first shown in [25], provides novel insights on the efficiency and effect of these design differentiations on suppressing ET tremor. Furthermore, an attempt is made to analyze the tremor properties for dual suppression methodology by simultaneously monitoring different arm points. Lastly, the efficiency of the proposed setup and derived protocol are extensively evaluated under different hand pose and motion scenarios performed by an ET-diagnosed volunteer. To the authors' best knowledge, this is the first time that such a soft-actuated exoskeleton has been tested on an ET patient.

It must be noted that the force controller utilized in this article is based on a PIDbased scheme. This conventional control algorithm has been documented for its efficiency in controlling PAM-enabled setups [26-29] and was selected as the most appropriate for the preliminary evaluation of the exoskeleton's tremor-suppressing abilities. Work on synthesis and further improvement of the control properties under active force and compliance control is currently in progress.

The rest of the article is structured as follows. In Section 2, the conceptual design of the hand exoskeleton is presented in detail, with highlighted overview of the implemented design alterations for investigating ET suppression on different regions of the lower arm. Section 3 deals with the force control methodology incorporated for both investigated cases, while Section 4 presents the setup prototype configurations and its utilized components. In Section 5, the results acquired from the experimental evaluation of the investigated setups on an ET-diagnosed volunteer are provided and analyzed. Finally, concluding remarks and commentary regarding future work are given in Section 6.

\section{Conceptual Design}

The structural challenge has been the development of an exoskeletal glove, which is capable of producing a resistive force on the fingertips while being manufactured with as few hard materials as possible and, thus, providing a light, comfortable, safe, lowconstruction-cost device. The base exoskeletal design (Figure 1) consists of a soft glove 
providing protection and attachment points on the forearm and hand, with 5 PAMs being strapped onto the forearm via connection caps, while tendons are adjusted between the PAMs and the caps that are placed on the user's fingers. Force sensors are properly placed inside the finger caps at the point of contact with the fingertips. It must be noted that the base design was conceptually derived for applications requiring tactile and kinesthetic feedback, with the purpose of investigating its general use in physiotherapeutic applications, for interaction with objects in artificial reality environments etc.

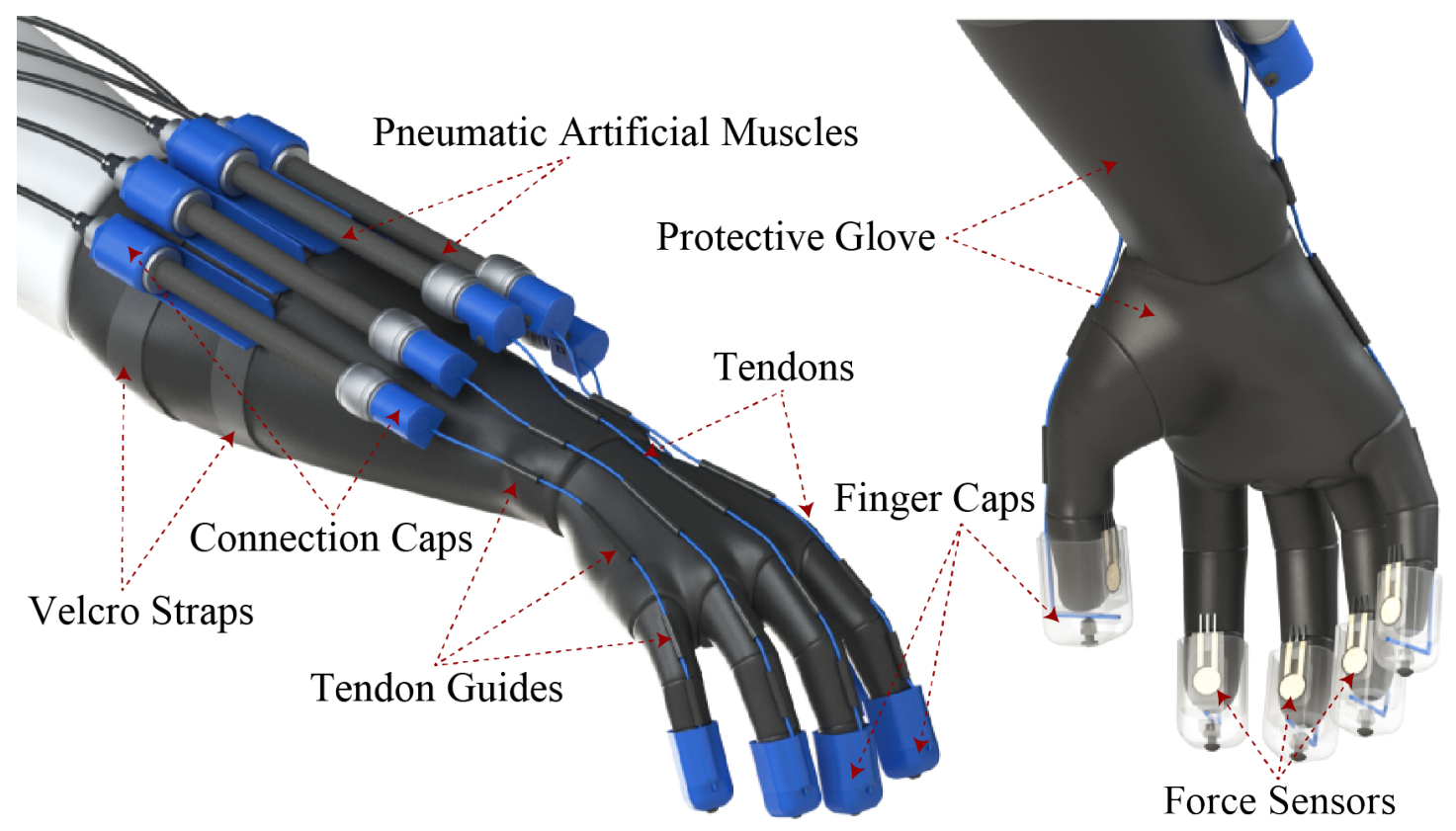

Figure 1. Overview of the exoskeletal glove base concept with highlighted main components.

For the purposes of investigating the tremor suppression capabilities of the exoskeleton glove and, in parallel, test the modularity and reconfigurability of the proposed concept, two subconfigurations were derived. Specifically, to evaluate the effect of applying kinesthetic forces on different parts of the hand of a tremor-diagnosed volunteer, the design was focused on a modified iteration for force application on the index finger (Figure 2A) and one with the resistive force applied on the metacarpal region (Figure 2B), hereafter mentioned as iterations (A) and (B), respectively.

Iteration (A) utilizes a single PAM connected to the user's index finger, in the same manner as described in Figure 1. Four accelerometers $a_{i, j}$ for $i=1,2, \ldots, 4$ and $j=x, y, z$ were properly placed on the distant phalanges of the index $\left(a_{1, j}\right)$ and thumb $\left(a_{2, j}\right)$ fingers, on the metacarpal $\left(a_{3, j}\right)$, and on the upper forearm $\left(a_{4, j}\right)$; their positioning was adjusted so as to be parallel to the respective skeletal frontal planes (Figure 2A). This arrangement was selected with the goal of assessing the tremor behavior on different fingers and parts of the lower arm, during local kinesthetic force application to a single finger.

Iteration (B) is focused on assessing the ET alterations on different lower-arm regions when resistive force is applied to the user's metacarpal area. To this goal, two PAMs were connected via tendons to a cylinder mechanism, which was firmly adjusted to the protective glove. The selection of the dual actuation was deemed necessary for ensuring symmetrical force application on the cylinder mechanism and increasing the system's bandwidth for handling the wrist's higher reactive forces. Specifically, the cylinder bears an internally a spring-based piston, which holds a force sensor on the piston's tip. Small metallic guide rods ensure the linear motion of the piston and the transfer of the pulling force on the metacarpal. This mechanism was selected for focusing and measuring the kinesthetic effect on the metacarpal region and avoiding the direct connection and subsequent effect on the fingers. Similarly, four, three-axis accelerometers $a_{i, j}$ were placed on the distant phalanges 
of the index $\left(a_{1, j}\right)$ and thumb $\left(a_{2, j}\right)$ fingers as well as on the lower $\left(a_{3, j}\right)$ and upper $\left(a_{4, j}\right)$ forearm, as depicted in Figure 2B.

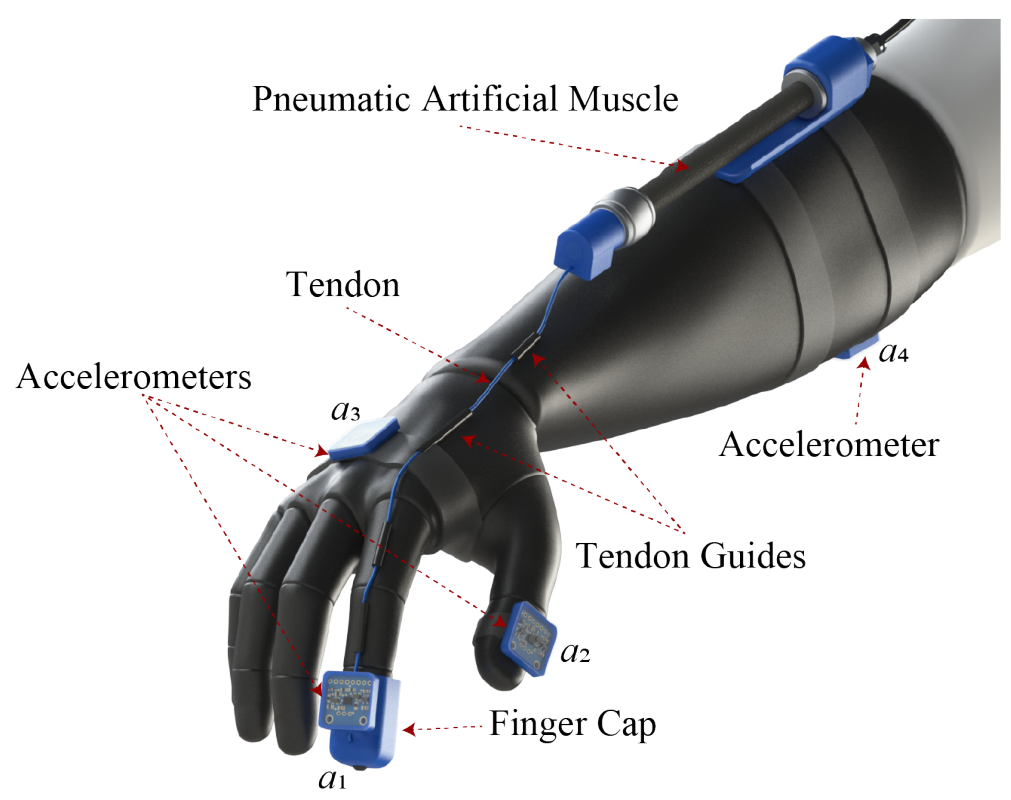

(A)

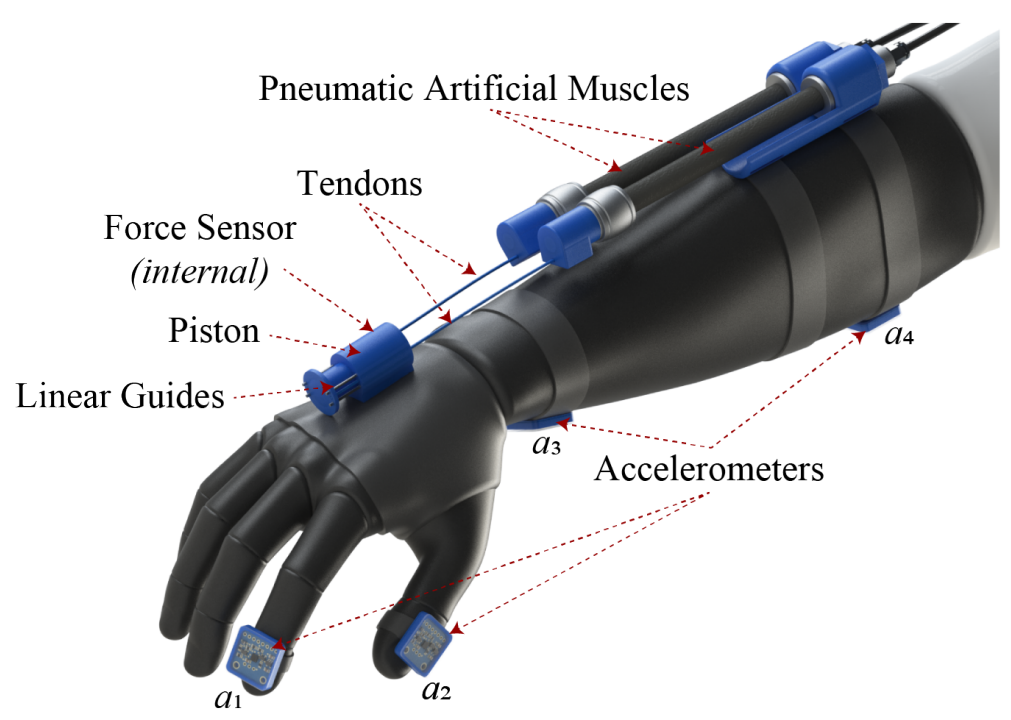

(B)

Figure 2. The exoskeletal glove concept for investigating tremor suppression via force application on the (A) index finger and (B) metacarpal region.

\section{Force Control Methodology}

For the design iterations (A) and (B) presented in the previous section, a force control scheme was synthesized for properly adjusting the pressure $P$ of air fed into the muscles and for controlling the applied resisting force $F$ on the target region. For the preliminary evaluation of the exoskeleton's tremor-suppressing abilities and given that both iterations were handled as Single-Input Single-Output (SISO) systems, a PID-based algorithm was selected as the most appropriate for this task due to its feasibility and easy implementation. Furthermore, PID provides a reliable solution when the system's parameters cannot be precisely estimated. Synthesis and implementation of model-based algorithms for further improving the control properties under active force and compliance control is considered part of future work. 
The scheme presented in Figure 3, features the feedback control action $P(t)$, which utilizes the weighted sum of three control parameters. Under the assumption of a sampling process with a sampling period $T_{S} \in R^{+}$, at sample $n \in Z^{+}$, the PID algorithm is mathematically formulated as in

$$
P(t)=K_{P} e(t)+K_{I} \int_{0}^{t} e(t) d \tau+K_{D} \frac{\mathrm{d} e(t)}{\mathrm{d} t},
$$

where $K_{P}, K_{I}$, and $K_{D}$ are the proportional, integral, and derivative gains, respectively. By an appropriate selection of the aforementioned control parameters, the controller's goal is to adjust the manipulated variable $P(t)$ and achieve minimization of the process' error signal $e(t)=F_{r e f}(t)-F(t)$, or equivalence between the set-point force $F_{r e f}$ and measured value $F$.

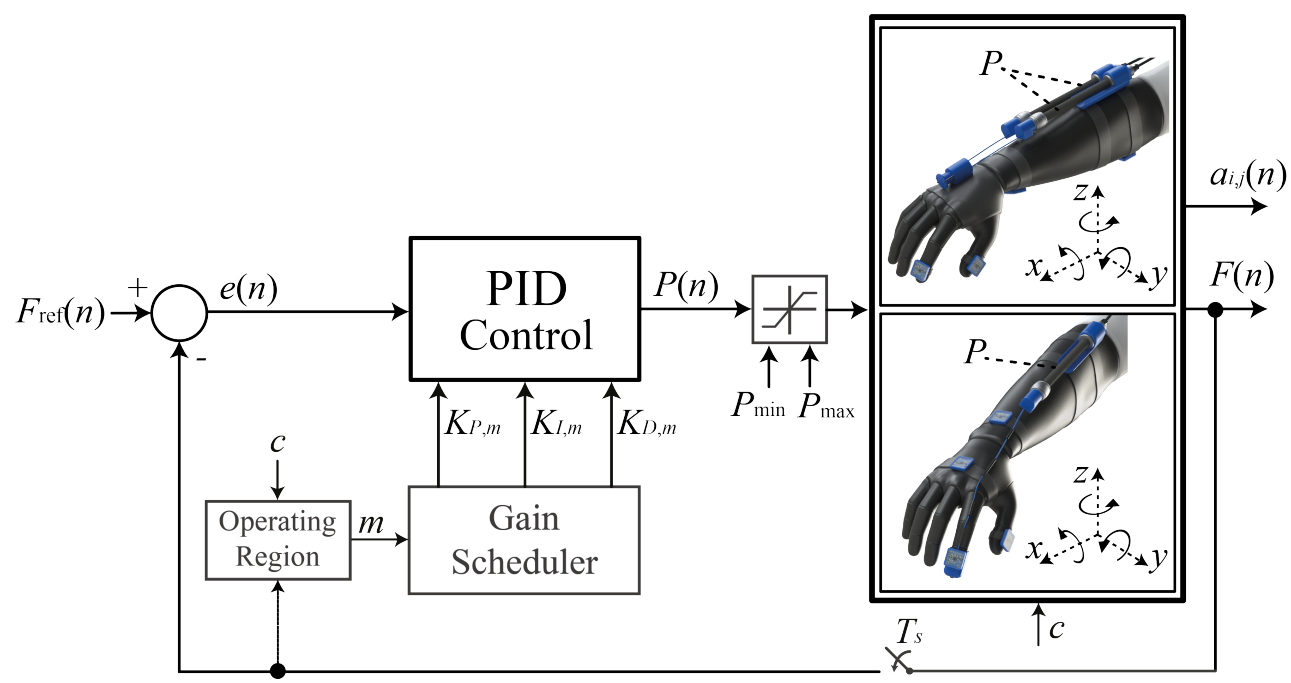

Figure 3. Block diagram of the incorporated control strategy.

The PAMs utilized in iterations (A) and (B) consist of a double helix aramid netting at a predetermined angle, covered by a neoprene threaded coating, thus, resulting in a tubelike formation. This dual-material formation is characterized by high nonlinearities that are presented below [30]:

- The viscoelastic properties of the neoprene wrapping;

- The friction phenomena between the aramid threads of the double helix weaving and the neoprene coating as well as the relative motion of the aramid threads themselves;

- the noncylindrical deformation of the tube edges during the PAM's inflation.

All the aforementioned properties result in complex hysteretic behavior during the PAM's operation. Furthermore, nonlinearities are also induced via the elastic components of the glove and soft tissue of the user's arm that can affect the overall performance of the setup. For the purposes of this investigation and given the Velcro-enabled muscle placement, the custom glove fitting, and the generally limited applied forces, the effect of such nonlinearities is assumed negligible, while their characterization and modeling is part of future iterations.

To counteract the PAM's intense nonlinearities, a gain scheduler is appropriately incorporated in the PID control structure, which has the ability to alter the control parameters according to the reference force level denoted by the signal $c$; it is used for switching between the reference and control values set for iterations (A) and (B), and according to the active region of operation, specified by the selected force $F$. The operating regions are defined during experimentation. The applied force was divided into smaller regions depending on the reference force $F_{r e f}$. Different points were defined experimentally via the Ziegler-Nichols frequency response method applied to each operating region and followed 
by additional fine-tuning through extensive experimental trials. In this case, an additional switching signal $m \in Z^{+}$is introduced that rules the switching values of the previously constant gains, as in the sequel:

$$
\left[\begin{array}{c}
K_{P}^{c} \\
K_{I}^{c} \\
K_{D}^{c}
\end{array}\right]=\left[\begin{array}{c}
K_{P, m}^{c} \\
K_{I, m}^{c} \\
K_{D, m}^{c}
\end{array}\right], \text { for } m=1,2, . ., M
$$

where $M \in Z^{+}$is the maximum number of operating regions. The discrete equation of the implemented gain-scheduled PID using the Backward Euler formula for the integration and derivative filter is presented in (3):

$$
C(z)=K_{P, m}^{c}+\frac{K_{I, m}^{c} T_{s} z}{z-1}+\frac{K_{D, m}^{c}(z-1)}{\left(1+T_{S}\right) z-1}
$$

Based on operational safety guidelines and the desirable control attributes, constraints are posed on the controllers' output $P(n)$ and set-point value $F_{r e f}(n)$, by setting minimum and maximum limits as specified in (4):

$$
\left[\begin{array}{c}
P_{\min }^{c} \\
F_{r e f, \min }^{c}
\end{array}\right] \leq\left[\begin{array}{c}
P^{c} \\
F_{r e f}^{c}
\end{array}\right] \leq\left[\begin{array}{c}
P_{\text {max }}^{c} \\
F_{r e f, \max }^{c}
\end{array}\right]
$$

\section{Prototype Setup Components}

\subsection{Exoskeletal Glove Setup}

The prototype setup of the exoskeletal glove in its base configuration is presented in Figure 4 with the main components highlighted. As previously mentioned, this configuration was developed and is currently utilized for its general use in physiotherapeutic applications and the study of kinesthetic interaction with objects in artificial reality environments. In the sequel, the description of the setup components will be focused on the investigated iterations (A) and (B).

Specifically, both iterations utilized a protective glove made from Velcro-friendly material, for attaching the PAMs via the 3D-printed connectors depicted in Figure 5A,B. For actuation purposes, iteration (A) utilized one Festo DMSP-10-120N-AM-CM Fluidic Muscle with $10 \mathrm{~mm}$ internal nominal diameter, $120 \mathrm{~mm}$ nominal length, $160 \mathrm{~g}$ weight, and $630 \mathrm{~N}$ maximum force output, while two muscles of the same properties were used in iteration (B). The nominal length of the PAMs was appropriately selected by taking into consideration the dimension restrictions of the skeletal structure, while the PAMs' placement played a crucial role in the exoskeleton's bandwidth, since it acts as a trade-off between the permitted joint motion and the forces acting on the target regions, considering that the utilized PAMs were operated between $0-8$ bar.

Additionally, one Festo VPPM-6F-L-1-F-0L10H-V1N-S1 proportional pressure regulator was utilized to control the pressure of the compressed air supplied into the single PAM for (A) and into the two PAMs for (B), while providing a pressure measurement via its integrated sensor. Moreover, one Flexiforce A201 sensor was incorporated in the finger cap (A) and the piston (B) for measuring the force exerted from the PAMs, while four Adafruit ADXL345 accelerometers were properly placed in (A) on the distant phalanges of the index and thumb, metacarpal, and upper forearm region-and in (B) on the distant phalanges of the index and thumb, and on the lower and upper forearm, as described and defined in the previous section. The control of the setup's operation, as well as the data acquisition, were achieved via a National Instruments USB-6215 and two Arduino Mega, while the setup's programming software was developed in National Instruments LabVIEW. 


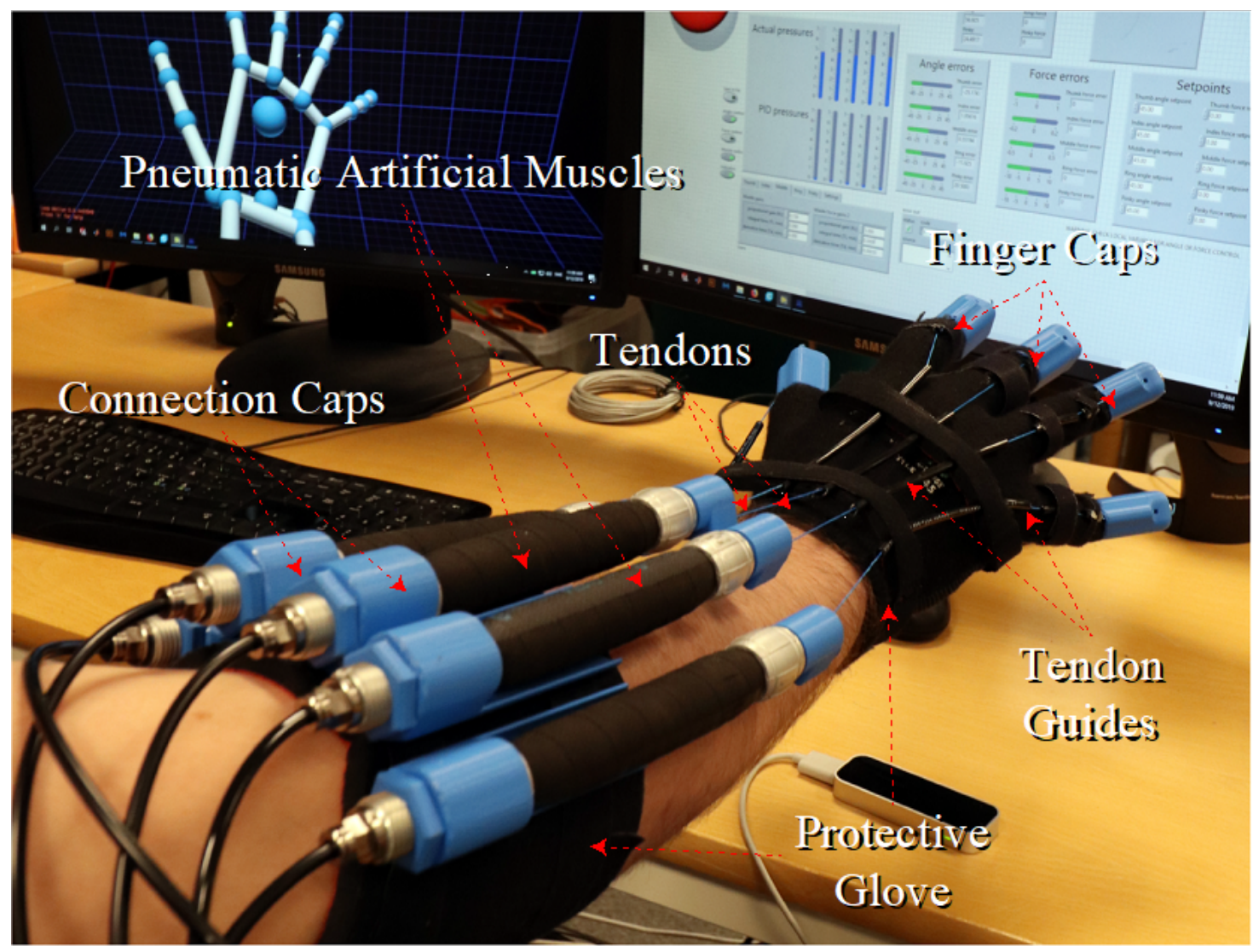

Figure 4. The exoskeletal glove base prototype.

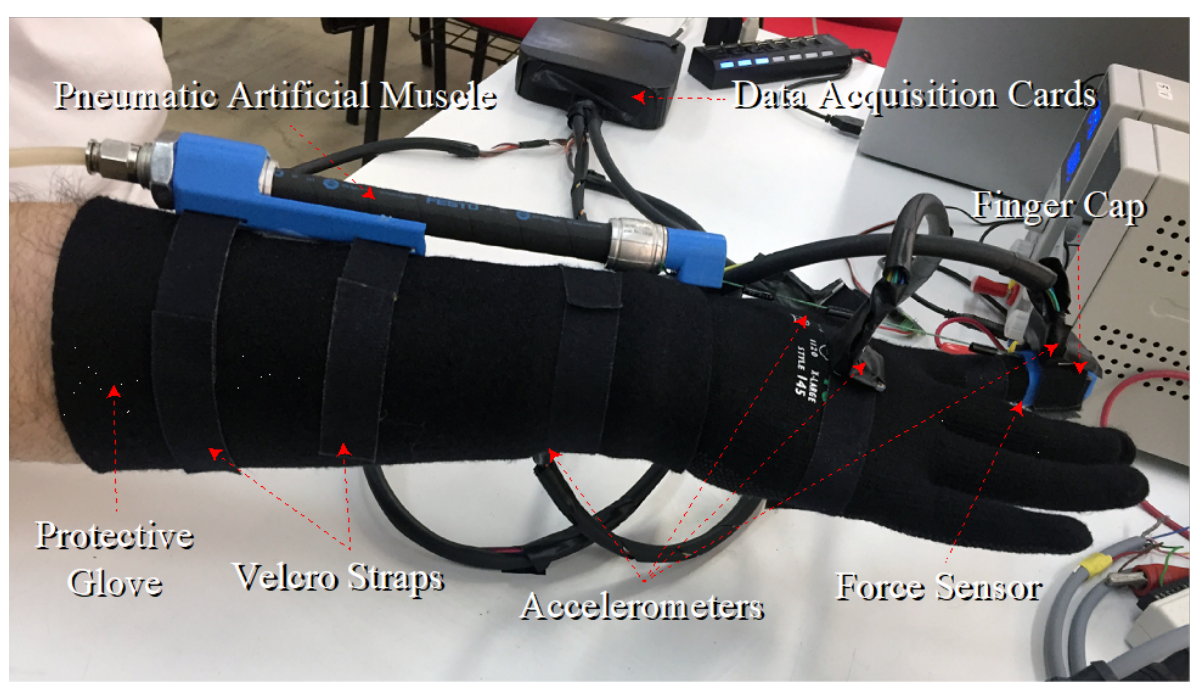

(A)

Figure 5. Cont. 


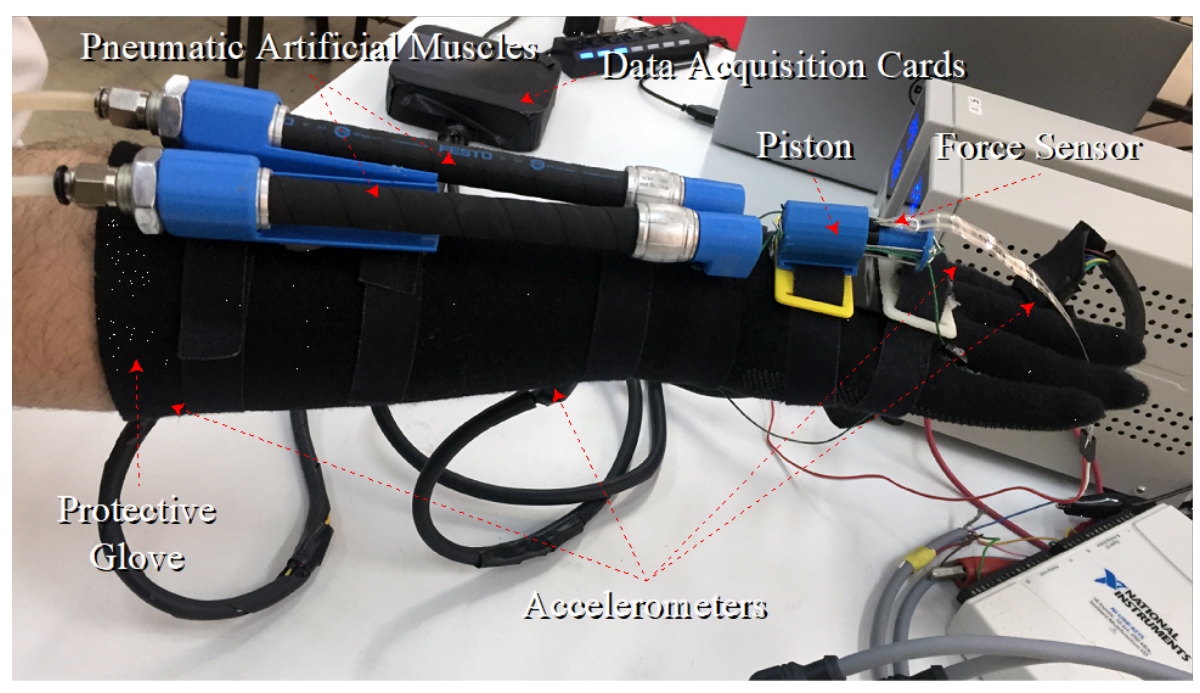

(B)

Figure 5. Overview of the exoskeletal glove prototype iterations for applying kinesthetic force to (A) the index finger and (B) the metacarpal region.

\subsection{Acceleration Signal Acquisition Setup}

In order to investigate the effect of utilizing tremor data from different parts of the hand as well as to have a tool to ensure the successful and safe data acquisition for results and performance evaluation, a signal acquisition device was developed. The device is based on the use of 4 accelerometers for enabling the extraction of hand-tremor information from multiple measurement points.

Specifically, the index and thumb were selected for their tactile dominance and kinematic difference, while the metacarpal and forearm were chosen to further analyze tremor origins and its propagating properties. Thus, for iteration (A), the accelerometers are placed each on the index, the thumb, the outer side of the metacarpal region, and on the lower part of the volunteer's forearm, as graphically shown in Figure 6; for iteration (B), they are placed each on the index, the thumb, and on the lower and upper part of the forearm. The placement of the accelerometers is properly stabilized via a protective glove and the use of Velcro straps. The standard coordinate systems are defined as presented in Figure 6, where the accelerometers are placed on the outer side of the hand and parallel to the transverse plane formed when the hand is in the extended position.

To be able to extract the necessary acceleration data, an acquisition device was developed for implementing the aforementioned conceptual approach. The device (Figure 7) consists of 4 ADXL345 3-axes accelerometers connected to two Arduino Mega Boards via SPI protocol. For iteration (A), the acceleration vector from each of the 4 accelerometers is defined as $a_{i, j}$, where $i=1,2, \ldots, 4$ for the accelerometer's placement on the index, the thumb, the metacarpal, and the forearm, respectively, and $j=x, y, z$ on the respective axis. For iteration (B), the acceleration vector from each of the 4 accelerometers is defined as $a_{i, j}$, where $i=1,2, \ldots, 4$ for the accelerometer's placement on the index, the thumb, and the lower and the upper forearm, respectively, and $j=x, y, z$ on the respective axis. The reason for utilizing two boards was for ensuring the highest possible sampling rate during the tremor recording sessions, enough for safely measuring the maximum ET frequencies. Double-sided Velcro straps are used for easy and comfortable adjustment of the accelerometers on the volunteer's hand, while minimizing the effect on the measurements during hand motions and maintaining the tactile ability of the person's fingertips.

Lastly, the Leap motion sensor displayed in Figure 4 was deemed unsuitable for use in the presented investigation, given its inability to continuously track the fingers in cases of patients with chronic tremor disorders, due to the increased finger stiffness and the resulting challenging hand pose, which the sensor failed to identify without inaccuracies and measurement gaps. 
Overall, the mechanical concept presented in its prototype form follows a patch-anduse logic, in which all equipment are easily installed via wear, patch, and plug actions. By avoiding the use of complex mechanisms comprising hard and heavy materials, the ease of installation and use is increased without lowering the durability factor that is of utmost importance for long hour uses. Specifically, the implementation approach led to two setups weighing approximately (A) $280 \mathrm{~g}$ and (B) $430 \mathrm{~g}$, while the base setup weights about $980 \mathrm{~g}$. In addition, the developed setups are characterized by a uniformly distributed weight, thus leading to minimum inertia and less actuator power used in order to counterbalance the gravity effects.

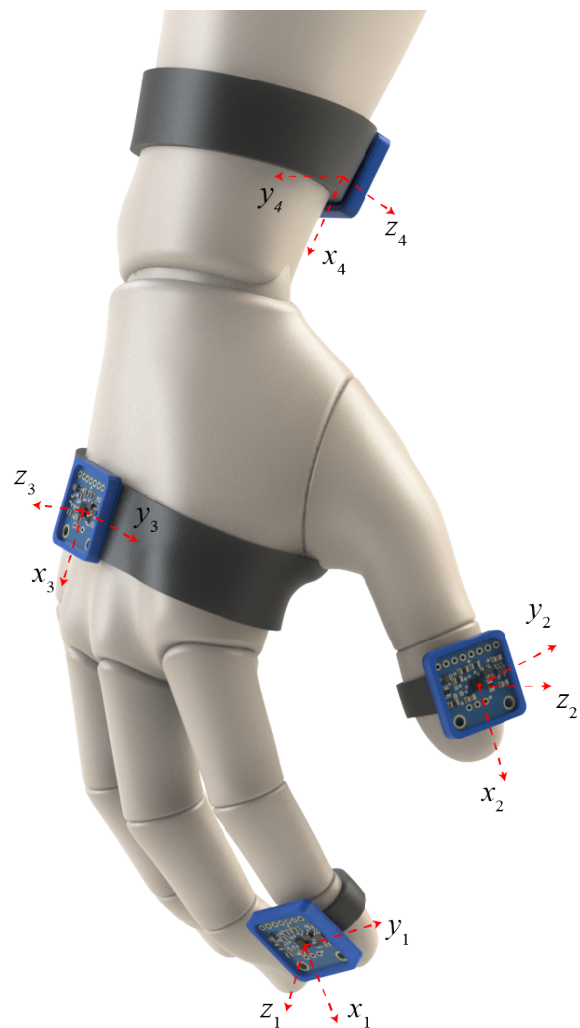

(A)

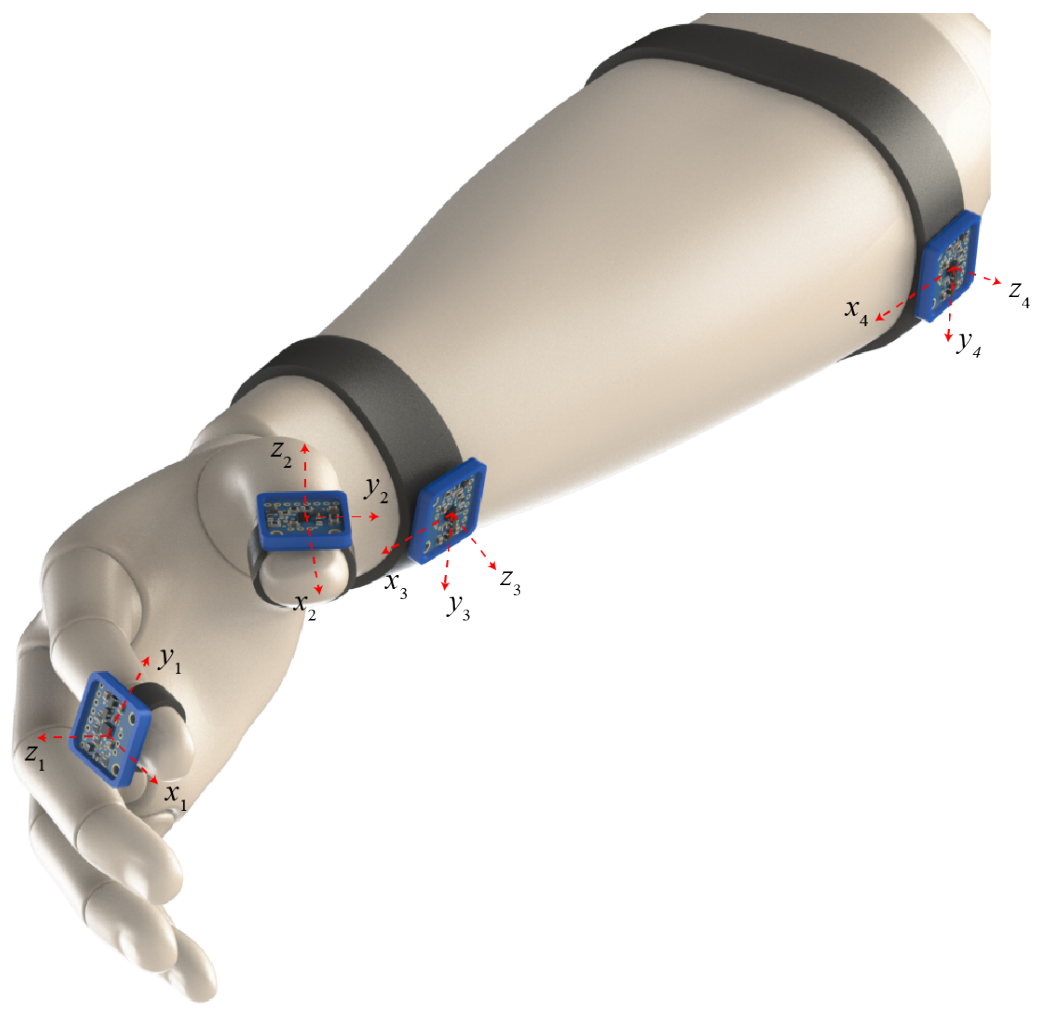

(B)

Figure 6. Graphical representation of the selected accelerometer placement points on the human hand for the presented (A) index and (B) metacarpal implementations of the exoskeletal glove.

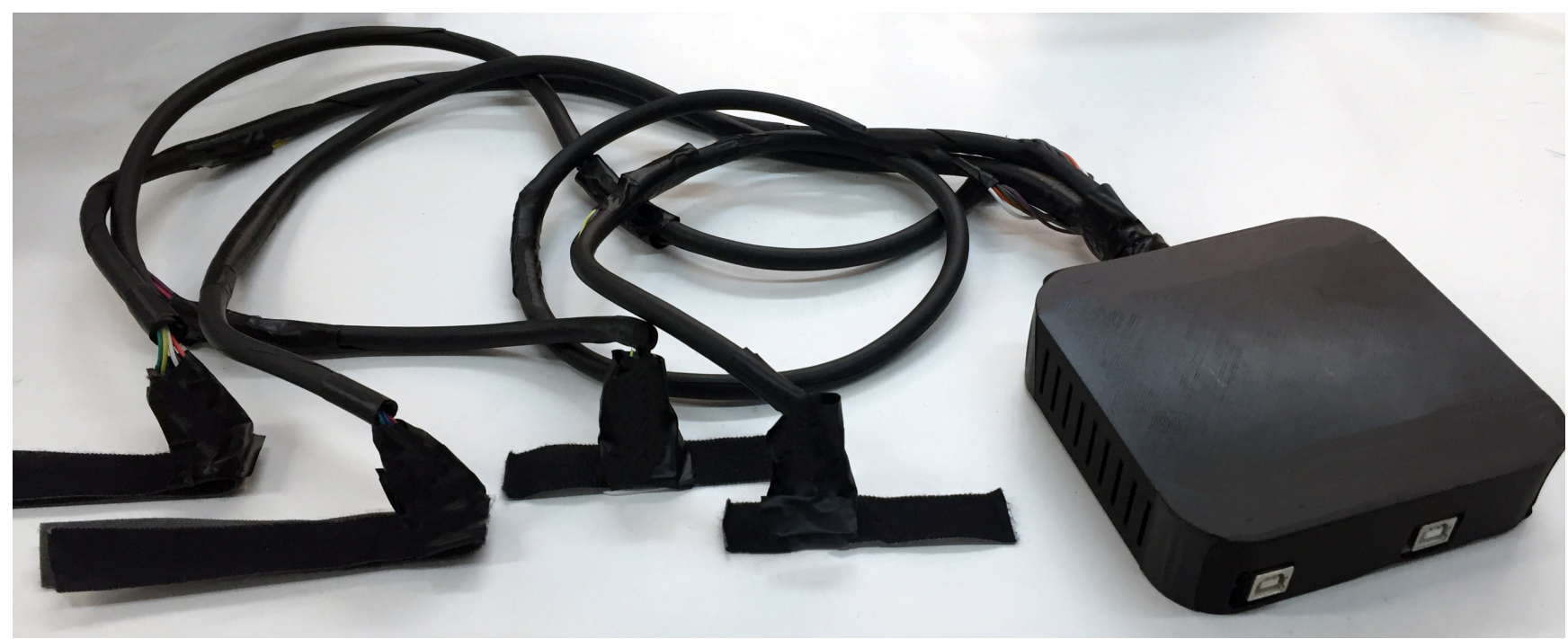

Figure 7. The accelerometer signal acquisition device. 


\section{Experimental Evaluation}

The accelerometer data extracted from the signal acquisition device include the signals of acceleration in $x, y, z$ axes as a function of time $t$ and are utilized to calculate the frequency spectrum of amplitude of the resultant acceleration vector of the measured signal for the extraction of the dominant tremor frequency. Indicatively, Figure 8A,B present the data acquired via (a) an ET-diagnosed patient and (b) a volunteer without tremor evidence. The acceleration measurements refer to the extended arm position for the index $I_{2}$. It is noted that for the amplitude spectrum plots in Figure 8, a high-pass filter was applied with a cutoff frequency of $0.5 \mathrm{~Hz}$ to avoid the DC artifact at $0 \mathrm{~Hz}$.
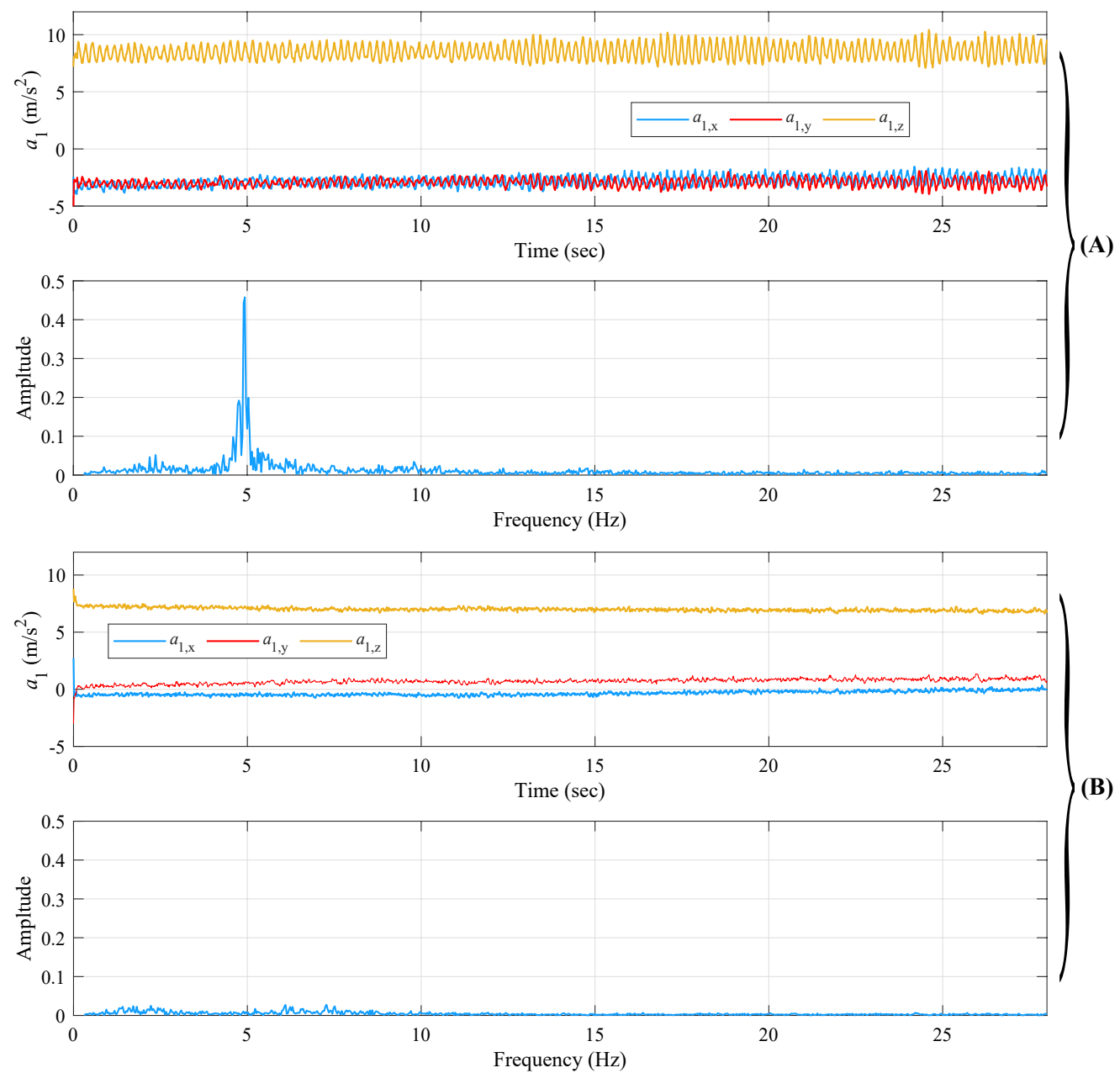

Figure 8. Indicative accelerometer measurements and respective frequency spectrum, as acquired from the index measurement point and the extended arm pose $I_{2}$ of (A) an ET-diagnosed patient and (B) a volunteer with no visible tremor evident.

From Figure 8A, the main extracted ET frequency for the specific measurement is easily distinguished and observed close to $4.93 \mathrm{~Hz}$, while the amplitude is 0.5. From Figure 8B, it is easily distinct how the volunteer keeps his arm extended without shaking significantly. However, as shown by the spectral diagrams, some small amplitude frequency peaks appear, the causes of which are attributed to the measurement noise from the accelerometer. Finally, the nontrembling volunteer's tremor frequency shows a significantly smaller amplitude in comparison to the tremor-diagnosed one, as expected.

For experimentally evaluating the ET suppression capabilities of the proposed prototypes (A) and (B), a 53-year-old, male, right-handed volunteer, diagnosed with chronic ET of 45 years, was selected. As noted in Table 1, although the volunteer receives medication for his condition, during the experiment procedure, he had not taken any pills in the last 
$12 \mathrm{~h}$. Prior to commencement of the presented experimental study, the volunteer was informed verbally and in writing about the purpose of the study, the experimental protocol, and his right to cancel his participation at any time.

Table 1. ET volunteer clinical profile.

\begin{tabular}{ll}
\hline Age & 53 \\
Sex & Male \\
Disease & Essential tremor \\
Years with tremor symptoms & 45 \\
Medication & Yes \\
Medication state during the experimental evaluation & OFF \\
Experimental area & Private \\
\hline
\end{tabular}

Initially, and before beginning the basic experimental procedure, we extracted the dominant tremor frequency of the ET participant's hand in 4 different hand positions/motions. In Table 2, the dominant tremor frequency observed from the participant during different hand motions is shown. The frequency from each of the 4 accelerometers of the signal acquisition device is defined as $f_{i, m}$, where $i=1,2, \ldots, 4$ for the accelerometer's placement on the index, the thumb, the metacarpal, and the forearm, respectively, and $m=1,2,3,4$ for the hand at resting position, at postural position, performing free motion, and performing motion while holding a spherical object, respectively.

All following experimental sequences began by mounting the setup on the volunteer's right hand, followed by the proper adjustment of the tendons while the volunteer's hand was in a straight position and the PAMs were fully contracted at the maximum permissible pressure. This adjustment ensured that the glove would not forcefully hyperextend the volunteer's fingers at any point, thus eliminating the potential risk of inducing extreme joint movements and injuries.

Initially, all experimental sequences involving the presented prototypes were executed under open-loop control of the kinesthetic force, for evaluating the setups' force limits and recording the effect of increasing the localized kinesthetic force on the volunteer's ET measurements from the four utilized accelerometers. Following the open-loop experiments, closed loop sequences involving the PID control scheme presented in Section 3 were performed to evaluate the controller's efficiency in tracking the setpoint value and the overall effect on the volunteer's ET characteristics.

Table 2. Dominant tremor frequencies in Hz observed for the ET-diagnosed participant.

\begin{tabular}{|c|c|c|c|c|c|c|c|c|c|c|c|c|c|c|c|}
\hline$f_{1,1}$ & $f_{1,2}$ & $f_{1,3}$ & $f_{1,4}$ & $f_{2,1}$ & $f_{2,2}$ & $f_{2,3}$ & $f_{2,4}$ & $f_{3,1}$ & $f_{3,2}$ & $f_{3,3}$ & $f_{3,4}$ & $f_{4,1}$ & $f_{4,2}$ & $f_{4,3}$ & $f_{4,4}$ \\
\hline 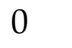 & 5.72 & 7.78 & 8.41 & 0 & 5.42 & 7.34 & 8.14 & 0 & 5.35 & 7.20 & 8.44 & 0 & 5.56 & 7.34 & 8.54 \\
\hline
\end{tabular}

The aggregated results regarding maximum ET frequency and amplitude reduction rates (\%) for iterations (A) and (B), under open-loop (OL) and closed-loop (CL) evaluations, are provided in Tables 3-11 and are discussed in detail in the next subsections. The naming utilized in the same Table involves the following:

- Iteration (A): the respective frequencies and amplitudes of the distant phalanges of the index $\left(f_{1, \text { max }}\right.$ and $\left.|a|_{1, \text { max }}\right)$ and thumb $\left(f_{2, \text { max }}\right.$ and $\left.|a|_{2, \text { max }}\right)$ fingers, the metacarpal $\left(f_{3, \max }\right.$ and $\left.|a|_{3, \max }\right)$, and the upper forearm $\left(f_{4, \max }\right.$ and $\left.|a|_{4, \max }\right)$.

- Iteration (B): the respective frequencies and amplitudes of the distant phalanges of the index $\left(f_{1, \text { max }}\right.$ and $\left.|a|_{1, \text { max }}\right)$ and thumb $\left(f_{2, \text { max }}\right.$ and $\left.|a|_{2, \text { max }}\right)$ fingers, the lower forearm $\left(f_{3, \max }\right.$ and $\left.|a|_{3, \max }\right)$, and the upper forearm $\left(f_{4, \max }\right.$ and $\left.|a|_{4, \max }\right)$.

\subsection{Prototype (A) Open-Loop Evaluation}

The open-loop evaluation of iteration (A) and the effect of applying resisting force on the index finger was performed via three experiments examining ET suppression 
for variable increases in pressure, while the volunteer's hand was in an extended pose. The results concerning the applied pressure $P$, the resulting force $F$, and the measurements from the four three-axis accelerometers are presented in Figures 9-11.

For the first open-loop experimental evaluation of iteration (A) and by observing Figure 9, the pressure follows an increasing and decreasing trend, reaching a maximum of 5.3 bar, while the force is alternating between 0 and $15 \mathrm{~N}$. The documented minor force alterations were also caused by minor involuntary reactions of the volunteer's hand during the pressure increase.

At time intervals when no force is exercised, i.e., $0-12 \mathrm{~s}$ and $32-41 \mathrm{~s}$, tremor is clearly visible in the volunteer's index and thumb measurements. Then, during time intervals 26-32 s and 54-64 s, where the applied $F$ starts to stabilize, a gradual decrease in ET amplitude and frequency is observed for all measurement points, as shown in Table 3 for the (A)-(OL) case. The maximal reduction was recorded on the metacarpal at $70 \%$ ET frequency with the respective reduction on the index straying at the lower level of $51 \%$, while ET amplitude was reduced at a maximal $75 \%$ on the index finger. Therefore, although the force from the PAM is exerted on the index finger, it indirectly affects the other parts of the lower arm in stabilizing their tremor. Such an effect could originate from the distribution of the force coefficient acting coincidentally to the bone structure, as well as the tendon contact forces through the guides.

The 2nd experiment-in addition to verifying repeatability—aims to emphasize the voluntary patient-induced occurrence of tremor as observed between 56-62 s. During this time, the patient bends the wrist downwards, enhancing the amplitude and frequency of tremor, as seen in Figure 10, resulting in a large decrease in ET amplitude of the index and thumb and an observable decrease on the metacarpal and forearm. Furthermore, the sharp and large increase in ET amplitude on the index and thumb, as well as the smaller but visible increase in the amplitude of the oscillation of the metacarpal as a result of the voluntary movement, denote the capability of the volunteer to activate/increase his tremor by performing short, voluntary movements, without necessarily tiring his hand out.

In the next experiment, $P$ was increased and held steady at its maximum value, i.e., 6 bar. As shown in Figure 11, $F$ is not stabilized around any point for a period longer than $2 \mathrm{~s}$. At the time between 12 and $28 \mathrm{~s}$, when the amplitude and frequency of the tremor on the two fingers is significantly reduced, increased tremor is noticeable on the forearm. As observed at the time of the experiment, a muscle at the top edge of the forearm started to pulsate. In more detail, $a_{4, x}$ and $a_{4, y}$ increase sharply from the 5 th $\mathrm{s}$ when $F$ starts to rise, and weaken from the 32nd s when $F$ begins to decrease, denoting a "tremor translation" behavior from the fingers to the rest of the hand. 

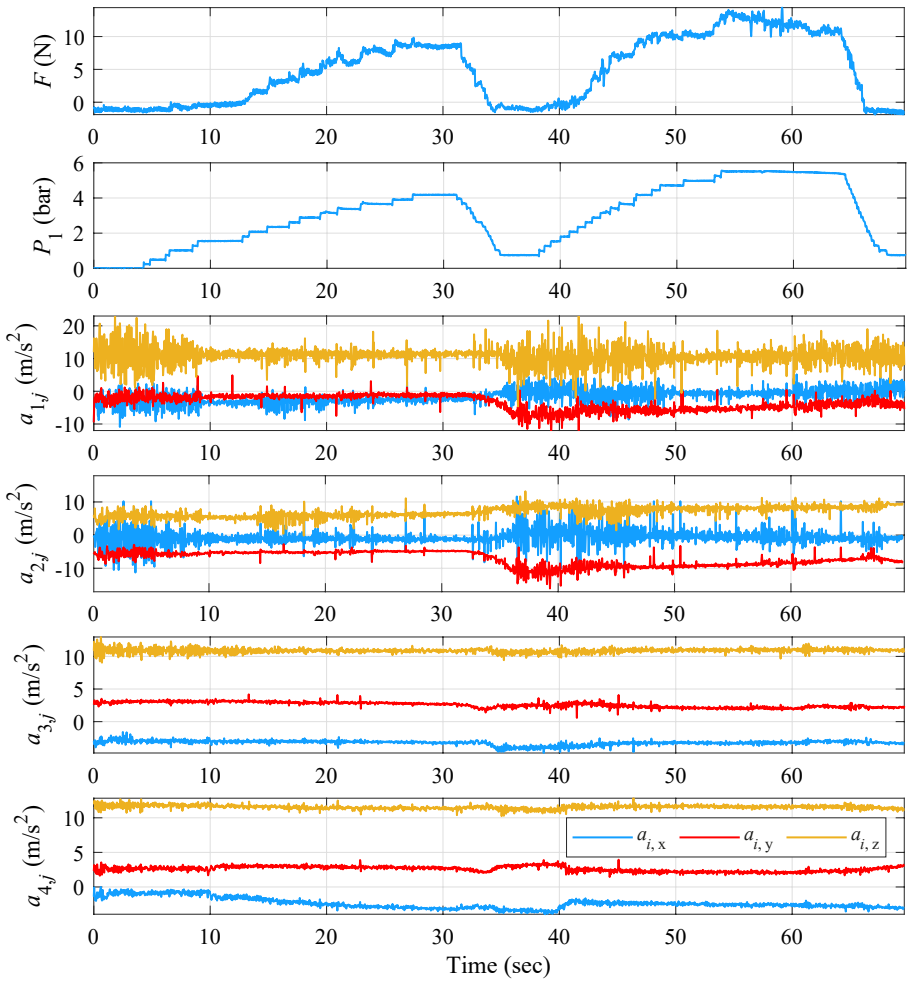

Figure 9. Experimental evaluation of iteration (A) and the effect of applying resisting force on the ET-diagnosed volunteer's index-finger under open-loop changes in applied pressure and while the hand is at extended pose; Exp. 1.
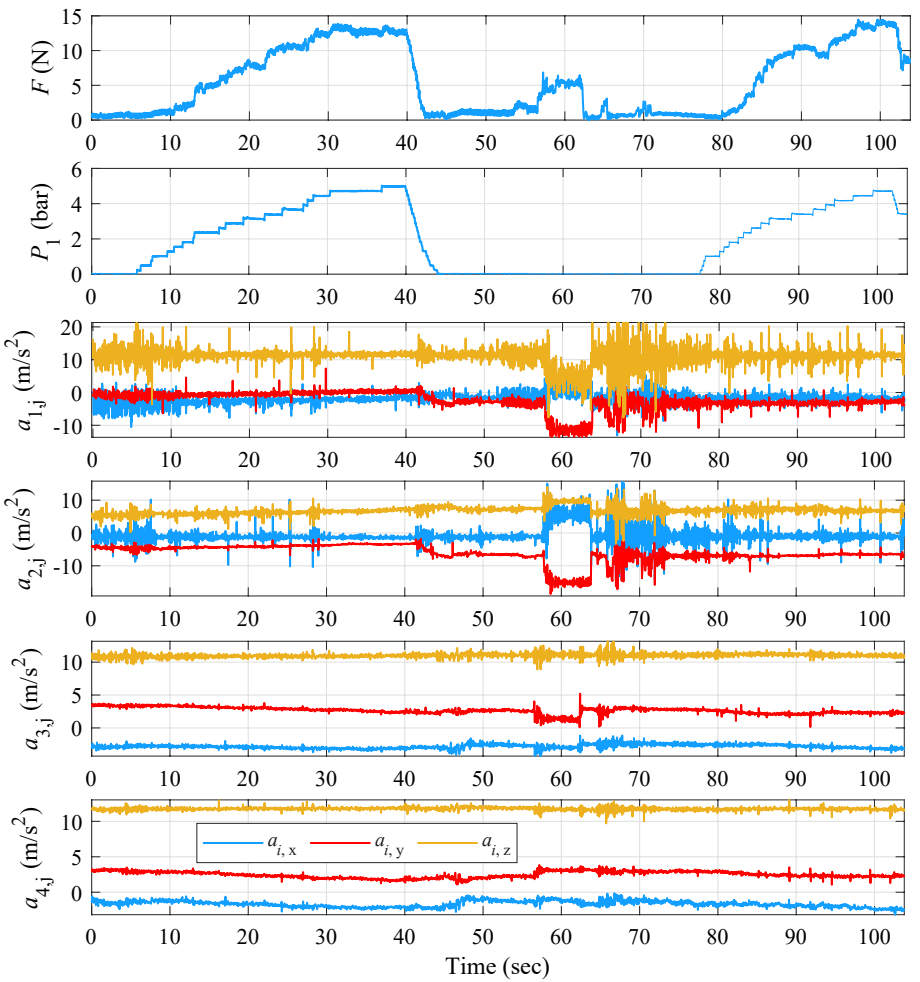

Figure 10. Experimental evaluation of iteration (A) and the effect of applying resisting force on the ET-diagnosed volunteer's index-finger under open-loop changes in applied pressure and while the hand is at extended pose and performing a voluntary movement; Exp. 2. 

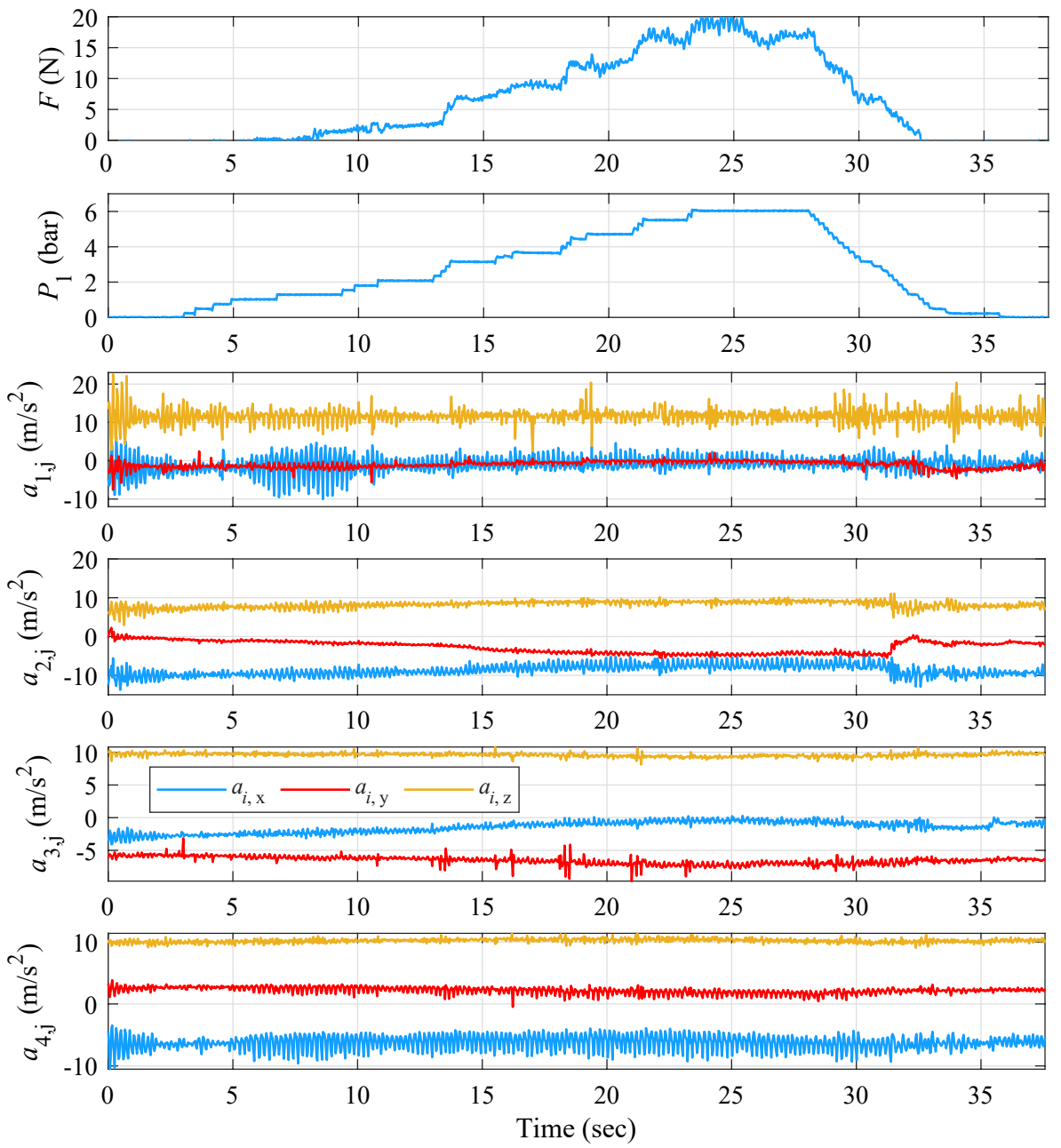

Figure 11. Experimental evaluation of iteration (A) and the effect of applying resisting force on the ET-diagnosed volunteer's index-finger under open-loop changes in applied pressure and while the hand is at extended pose. Tremor translation circumstance; Exp. 3.

By observing Table 3 , the largest decrease in $f_{1}$ is seen on the $\mathrm{z}$ axis with an average of $40 \%$, slightly higher than the other two axes. The largest decrease at the $\mathrm{x}$ and $\mathrm{y}$ axes appears in experiment 1 and amounts to 65 and $47 \%$, respectively; in experiment $2, f_{1, z}$ reaches its maximal reduction rate with $52 \%$. The largest rate of frequency reduction on the index is observed in experiment 1 with $51 \%$. On the thumb, the largest frequency reduction is achieved on the y axis with $44 \%$ and mainly in experiment 2 with $64 \%$. On the metacarpal, the maximum reduction is observed on the $\mathrm{z}$ axis with $77 \%$ with a significant difference from the other two axes. Experiment 2 has the optimum rate of tremor frequency reduction with $70 \%$. On the forearm, the maximum ET frequency decrease is detected on the y axis with $50 \%$, but close to the other two axes. Similarly to the metacarpal, experiment 2 provides the optimal frequency reduction rate of $62 \%$.

Table 3. ET frequency reduction rates during the Prototype (A) Open-Loop evaluation.

\begin{tabular}{lllllllllllllllll}
\hline Exp. & $f_{1, x}$ & $f_{1, y}$ & $f_{1, z}$ & $f_{1}$ & $f_{2, x}$ & $f_{2, y}$ & $f_{2, z}$ & $f_{2}$ & $f_{3, x}$ & $f_{3, y}$ & $f_{3, z}$ & $f_{3}$ & $f_{4, x}$ & $f_{4, y}$ & $f_{4, z}$ & $f_{4}$ \\
\hline 1 & 65 & 47 & 42 & 51 & 35 & 47 & 42 & 41 & 47 & 45 & 75 & 56 & 47 & 47 & 77 & 57 \\
2 & 38 & 40 & 52 & 43 & 26 & 64 & 38 & 43 & 69 & 63 & 78 & 70 & 67 & 62 & 0 & 64 \\
3 & 17 & 20 & 27 & 21 & 17 & 21 & 30 & 23 & 20 & 30 & 77 & 42 & 20 & 20 & 57 & 32 \\
\hline Av. & 35 & 36 & 40 & 38 & 26 & 44 & 33 & 36 & 45 & 46 & 77 & 56 & 45 & 50 & 45 & 51 \\
\hline
\end{tabular}


Looking at Table 4, the largest decrease in ET amplitude on the index is observed at the $y$ axis with an average percentage of $70 \%$. The largest decrease on the $x$ axis is observed in experiment 1 and amounts to $94 \%$, and on the $y$ and $z$ axes in experiment 2 with $86 \%$ and $54 \%$, respectively. The largest rate of reduction on the index is found in experiment 1 with $75 \%$. On the thumb, the greatest reduction in tremor amplitude lies on the $\mathrm{x}$ axis with $76 \%$, with significant differences to the other two axes. The largest decrease on the $x$ axis is observed in experiment 1 and amounts to the high $99 \%$. The largest decrease on the thumb is found in experiment 2 with $71 \%$. On the metacarpal area, the maximum reduction on the $\mathrm{x}$ axis is $50 \%$, while the reduction rates on the $\mathrm{y}$ and $\mathrm{z}$ axes are low. The largest tremor amplitude decrease appears in experiment 2 and amounts to $79 \%$ on the $\mathrm{x}$ axis. On the forearm, the maximum decrease is detected on the $\mathrm{x}$ axis and reaches up to $35 \%$. The greatest reduction rate is observed in experiment 2 with $57 \%$ on the $x$ axis. It should be noted that for the export of the mean reduction rates on the forearm, the percentages of the $3_{r d}$ experiment were not taken into account, as there are increased rates on the $\mathrm{x}$ axis. Regarding this experiment, and especially for the forearm region, from Table 4, an increase in ET is noticeable on the $\mathrm{x}$ axis by $42 \%$, confirming the "tremor translation" observation.

Table 4. ET amplitude reduction rates during the Prototype (A) Open-Loop evaluation.

\begin{tabular}{|c|c|c|c|c|c|c|c|c|c|c|c|c|c|c|c|c|}
\hline Exp. & $a_{1, x}$ & $a_{1, y}$ & $a_{1, z}$ & $a_{1}$ & $a_{2, x}$ & $a_{2, y}$ & $a_{2, z}$ & $a_{2}$ & $a_{3, x}$ & $a_{3, y}$ & $a_{3, z}$ & $a_{3}$ & $a_{4, x}$ & $a_{4, y}$ & $a_{4, z}$ & $a_{4}$ \\
\hline 1 & 94 & 78 & 53 & 75 & 99 & 29 & 36 & 55 & 20 & 11 & 10 & 14 & 13 & 7 & 6 & 9 \\
\hline 2 & 30 & 86 & 54 & 57 & 94 & 66 & 53 & 71 & 79 & 28 & 10 & 39 & 57 & 21 & 6 & 28 \\
\hline 3 & 58 & 46 & 46 & 50 & 34 & 63 & 1 & 33 & 52 & 19 & 1 & 24 & -42 & 11 & 1 & -10 \\
\hline Av. & 61 & 70 & 51 & 74 & 76 & 53 & 30 & 53 & 50 & 19 & 7 & 26 & 35 & 13 & 4 & 12 \\
\hline
\end{tabular}

\subsection{Prototype (A) Closed-Loop Evaluation}

The closed-loop evaluation of iteration (A) was initiated with a set-point reference force value set at approximately $6 \mathrm{~N}$, as obtained from (A)-(OL) as a suitable force level for effective ET reduction, while the volunteer's hand was in the relaxed state. The gain scheduler's control parameters $K_{P}, K_{I}$, and $K_{D}$ were tuned via the Ziegler-Nichols frequency response method applied on each operating region and followed by additional fine-tuning through extensive experimental trials. The aforementioned parameters are presented in Table 5 .

Table 5. PID parameters for the Prototype (A) Closed-Loop control scenario.

\begin{tabular}{lllll}
\hline $\mathbf{m}$ & $\begin{array}{l}\text { Operating } \\
\text { Regions [N] }\end{array}$ & $\boldsymbol{K}_{\boldsymbol{P}, \boldsymbol{m}}$ & $\boldsymbol{K}_{\boldsymbol{I}, \boldsymbol{m}}$ & $\boldsymbol{K}_{\boldsymbol{D}, \boldsymbol{m}}$ \\
\hline 1 & $F \in[0,2]$ & 1.00 & 0.10 & 0.02 \\
2 & $F \in(2,4]$ & 1.80 & 0.32 & 0.02 \\
3 & $F \in(4,6]$ & 2.40 & 0.40 & 0.12 \\
4 & $F \in(6,8]$ & 1.56 & 0.14 & 0.05 \\
\hline
\end{tabular}

From the results presented in Figure 12, it is observable that the index and thumb are initially characterized by a high ET amplitude and frequency before enabling the controller. A significant decrease in index ET amplitude can be observed in all measurement points for the time interval where the force approaches the reference signal. A similar decrease in both amplitude and frequency is also observed on the thumb. On the metacarpal region and at the upper forearm, a similar behavior is noticed, with an important decrease in ET amplitude and frequency when stabilizing the force around the reference signal. Then, the ET symptoms enhance again when disabling the controller at approximately $27.5 \mathrm{~s}$. In addition, the responses produced via the PID scheme are characterized by smooth control effort alterations, as the gain scheduler switches between neighboring regions, which results in very small variations from the reference signal. 

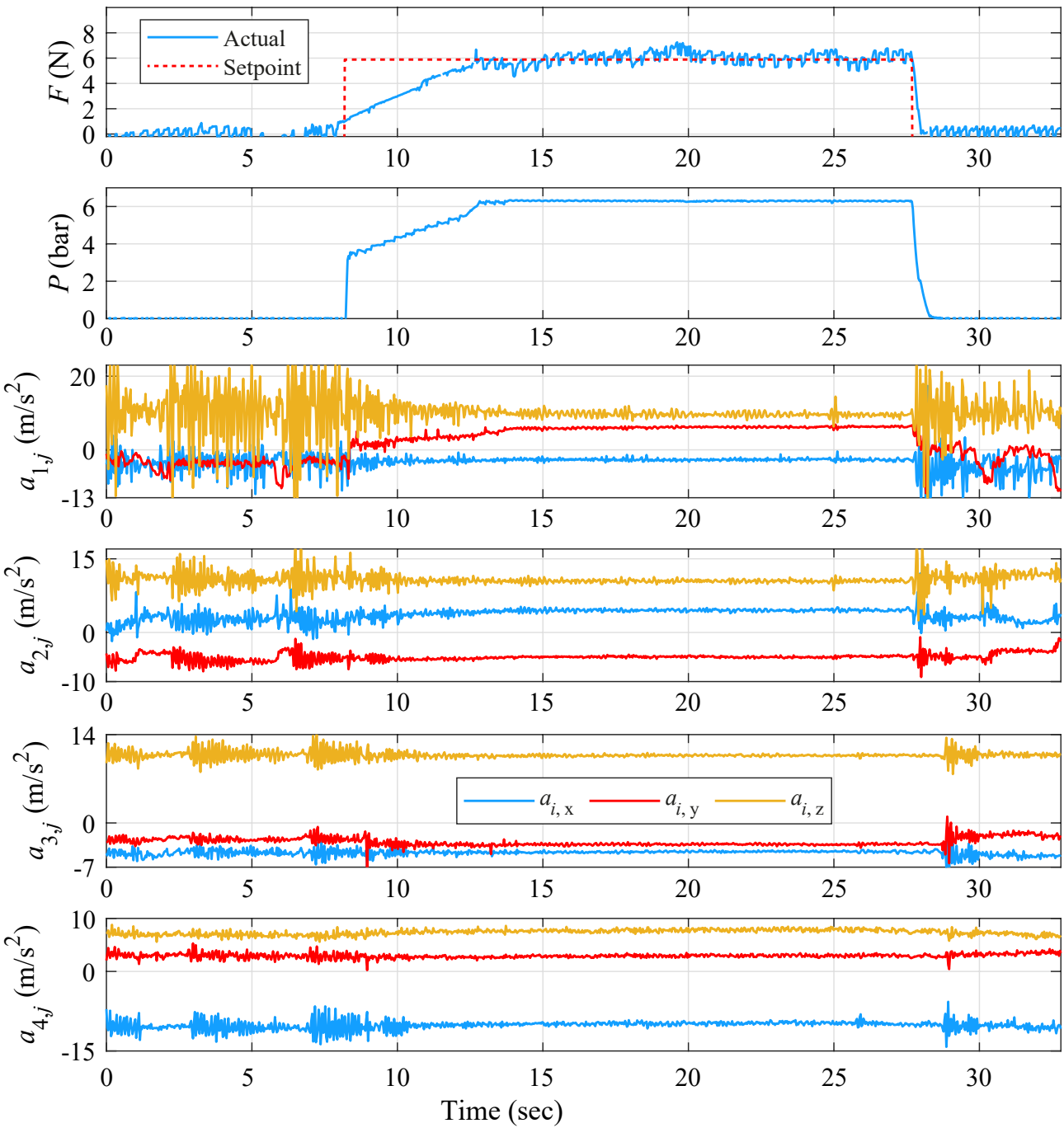

Figure 12. Experimental evaluation of iteration (A) and the effect of applying resisting force to the ET-diagnosed volunteer's index-finger under closed-loop reference in applied pressure and while the hand is at extended pose; Exp. 1.

Two additional experiments were performed while in closed-loop operation, where the controller was activated in the presence of ET, the volunteer was asked to bend his fingers until they touched a spherical object, and then to return them to their original position.

From the results presented in Figure 13, it is clearly observed that until the first $5 \mathrm{~s}$, the controller is turned off. The controller is activated from 5-41 s and from $60 \mathrm{~s}$ to the end of the trial. For the total duration of the experiment and throughout the volunteer's motion, the controller was tuned to provide smooth behavior with absence of abrupt alterations in pressure, although characterized by a slower convergence of about 4 sec per activation. At the activation intervals of 5-41 s and 60-95 s, where the controller attempts to track the force set-point despite the volunteer's movements, a noticeable reduction in the ET amplitude and frequency during the bending of the hand can be observed for all four measurement points, demonstrating the efficiency of the proposed solution. The overall force remains in small values ranging from approx. 1 to $4 \mathrm{~N}$, which do not significantly constrain the volunteer's finger mobility. Interestingly, at the metacarpal and upper forearm, the ET amplitude is not increased after the controller is deactivated at $41 \mathrm{~s}$. Possibly, a "stimulus" from the setup was enough to reduce the tremor's amplitude in those regions and continue this effect until the next occurrence. 

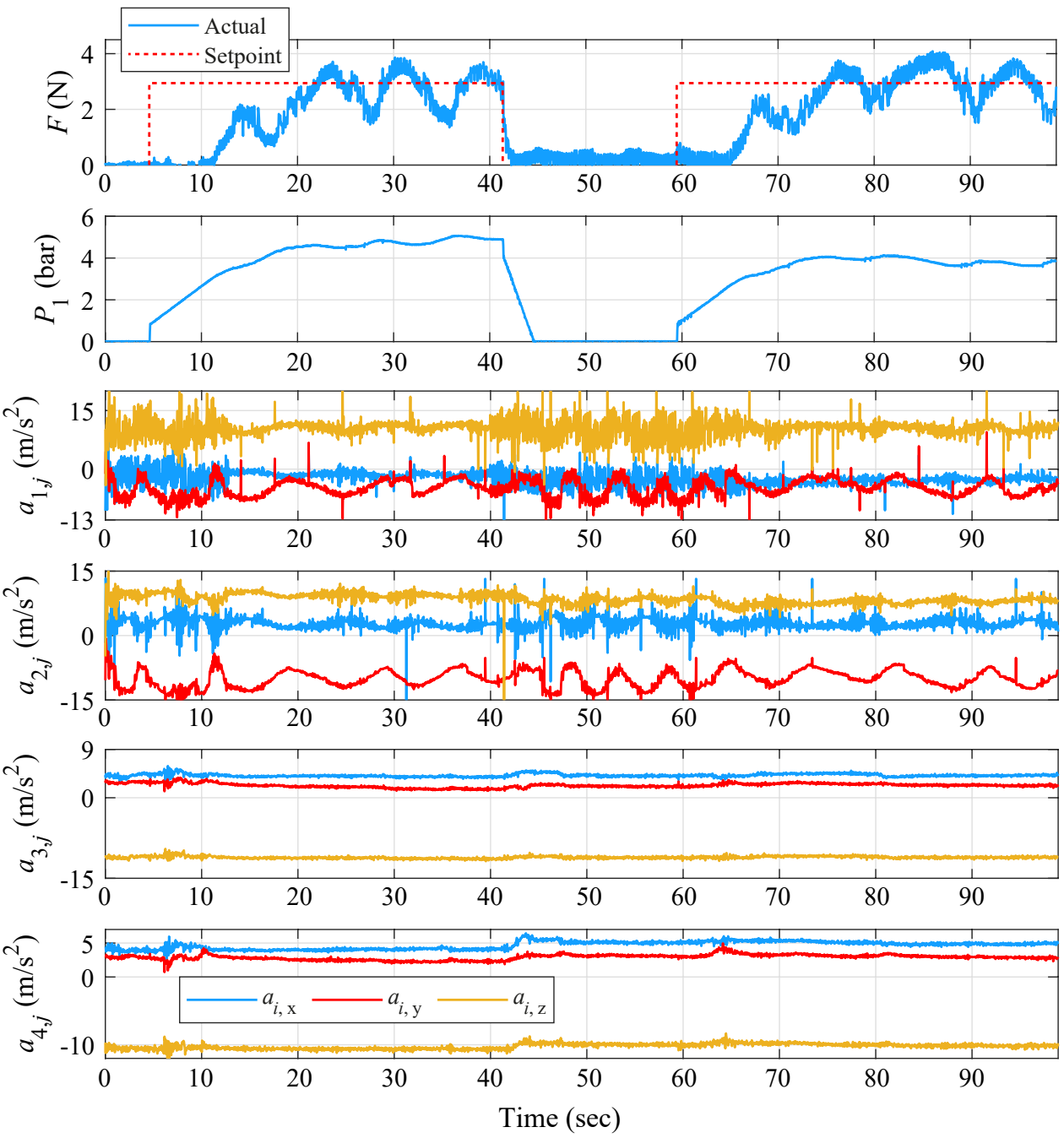

Figure 13. Experimental evaluation of iteration (A) and the effect of applying resisting force to the ET-diagnosed volunteer's index finger under closed-loop reference in applied pressure, while performing finger-bending motions that finish with touching a spherical object; Exp. 2.

The 3rd experiment describes the behavior of the suppression system when applying a more "counteractive" controller with increased proportional gain $K_{p}(3-33 \mathrm{~s})$ and a less "reactive" one with reduced $K_{p}(49-93 \mathrm{~s})$. It is noted that the $K_{i}$ and $K_{d}$ gains are tuned to responses that were satisfied by the convergence and oscillation characteristics, and therefore remain constant. As can be observed from $P(t)$ and $F(t)$ diagrams in Figure 14 and during the first controller activation, in order for $F$ on the index to approach the reference signal set at $5.5 \mathrm{~N}$, the pressure actuation is more direct and steep than during the second activation. So, even though the force reaches the setpoint faster than the first activation, the steady-state error is substantially greater.

Simultaneously, for both the controller activation periods and the tremor suppression on index and thumb fingers, the tremor amplitude on the metacarpal area and on the forearm increased. This "tremor translation" phenomenon was met once again for the open-loop case of iteration A.

According to Table 6, the largest average decrease in $f_{1}$ appears on the y axis with $47 \%$, while the $x$ and $z$ axes have average values of 36 and $28 \%$, respectively. The largest decrease on the $x$ axis appears in experiments 1 and 2 with $38 \%$, and on the $y$ and $z$ axes in experiment 2 with $51 \%$ and $37 \%$, respectively. The largest reduction rate on the index is met in experiment 2 with a rate of $42 \%$. On the thumb, as on the index, the largest frequency reduction is achieved on the $y$ axis with $65 \%$, but this time with a significant 
difference to the other two axes. The largest ET frequency reduction rate is observed in experiment 1 on the $y$ axis and amounts to $73 \%$. At the metacarpal area, the maximum reduction is observed on the $x$ axis with $71 \%$. The largest decrease on the $x$ axis is observed in experiment 2 with $73 \%$, while experiment 1 follows closely with $72 \%$. On the y axis, the maximum reduction percentage is found in experiment 2 with $62 \%$ and on the $\mathrm{z}$ axis again in the 2 nd experiment with $78 \%$. It is noted that experiment 1 has the optimum rate of tremor frequency reduction with $66 \%$. On the forearm and in experiments 1 and 2, the maximum reduction is detected on the $x$ axis at a rate of $67 \%$. The optimal reduction on the $x$ axis is observed in experiment 2 at $70 \%$. In experiment 3 , which concerns the "tremor translation" situation, a significant frequency increase is noticed at $f_{4, z}$, at a rate of $77 \%$. In general, for the case of experiment 3 , there is an average increase in tremor frequency of $29 \%$.
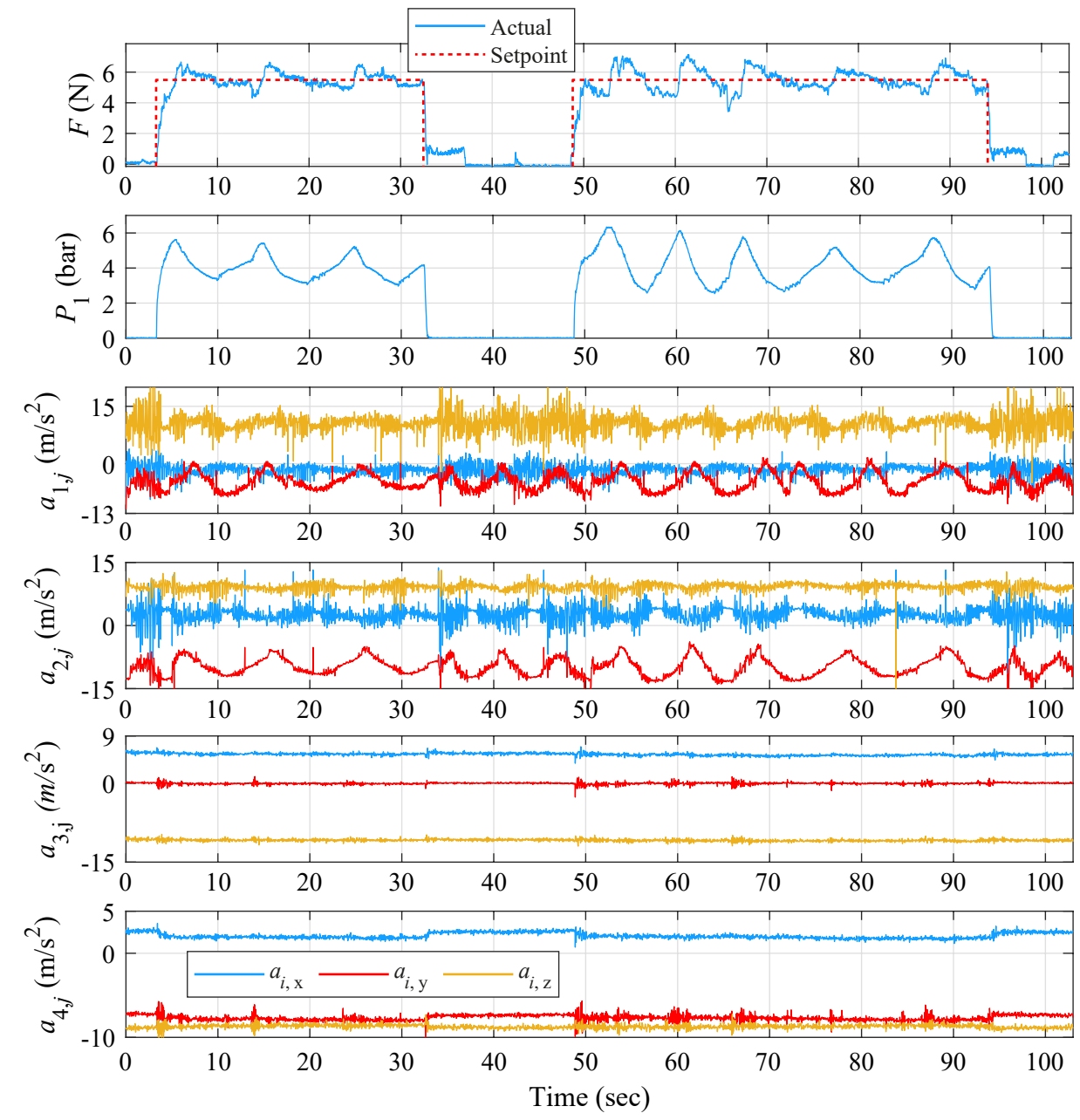

Figure 14. Experimental evaluation of iteration (B) and the effect of applying resisting force on the ET-diagnosed volunteer's metacarpal under open-loop changes in applied pressure and while the hand is at extended pose; Exp. 3.

Table 6. ET frequency reduction rates during the Prototype (A) Closed-Loop evaluation.

\begin{tabular}{lllllllllllllllll}
\hline Exp. & $f_{1, x}$ & $f_{1, y}$ & $f_{1, z}$ & $f_{1}$ & $f_{2, x}$ & $f_{2, y}$ & $f_{2, z}$ & $f_{2}$ & $f_{3, x}$ & $f_{3, y}$ & $f_{3, z}$ & $f_{3}$ & $f_{4, x}$ & $f_{4, y}$ & $f_{4, z}$ & $f_{4}$ \\
\hline 1 & 38 & 44 & 22 & 35 & 40 & 73 & 50 & 54 & 72 & 48 & 78 & 66 & 70 & 40 & 59 & 56 \\
2 & 38 & 51 & 37 & 42 & 21 & 68 & 44 & 44 & 73 & 62 & 0 & 45 & 74 & 63 & 72 & 70 \\
3 & 32 & 45 & 26 & 34 & 28 & 53 & 34 & 38 & 68 & 47 & 0 & 38 & 56 & -65 & -77 & -29 \\
\hline Av. & 36 & 47 & 28 & 37 & 30 & 65 & 43 & 45 & 71 & 52 & 26 & 50 & 67 & 51 & 44 & 63 \\
\hline
\end{tabular}


According to Table 7, the largest average decrease on the index ET amplitude is observed on the $\mathrm{x}$ axis with $88 \%$. The largest decrease on the $\mathrm{x}$ and $\mathrm{y}$ axes is observed in experiment 3 with $91 \%$ and $79 \%$, and on the $\mathrm{z}$ axis, in experiment 1 with $58 \%$. The largest rate of reduction on the index is found in experiment 3 with the quite high percentage of $75 \%$. On the thumb, the largest reduction on tremor amplitude is achieved on the y axis with $53 \%$, with only a slight difference to the $\mathrm{x}$ axis. The largest decrease on the $\mathrm{x}$ axis is observed in experiment 2 with $75 \%$, and on the $y$ and $z$ axes in experiment 1 with $74 \%$ and $42 \%$, respectively. In addition, the largest decrease in thumb is found in experiment 2 with a rate of $49 \%$. For the metacarpal, the maximum decrease occurs on the $\mathrm{x}$ axis with $43 \%$. The largest ET amplitude decrease for the three axes is observed in experiment 2. It is perceived that experiment 2 has the optimum tremor amplitude reduction rate with the not so high $35 \%$. It is also important to note that during experiment 3 , where the "tremor transition" phenomenon appeared, ET amplitude on $x$ axis almost doubled. For the forearm, the maximum reduction is detected on the y axis with $39 \%$, while the reduction rates on the $x$ and $z$ axes are also quite low at 34 and $16 \%$, respectively. The largest decrease on the $x$ axis is observed in experiment 2 with $36 \%$. Thus, experiment 2 appears to be the most effective with an average reduction rate of $35 \%$. It should be mentioned that in experiment 3 and for the "tremor translation" situation, there is an increase in tremor amplitude on all 3 axes with corresponding percentages of 83,23 , and $37 \%$, respectively.

Table 7. ET amplitude reduction rates during the Prototype (A) Open-Loop evaluation.

\begin{tabular}{|c|c|c|c|c|c|c|c|c|c|c|c|c|c|c|c|c|}
\hline Exp. & $a_{1, x}$ & $a_{1, y}$ & $a_{1, z}$ & $a_{1}$ & $a_{2, x}$ & $a_{2, y}$ & $a_{2, z}$ & $a_{2}$ & $a_{3, x}$ & $a_{3, y}$ & $a_{3, z}$ & $a_{3}$ & $a_{4, x}$ & $a_{4, y}$ & $a_{4, z}$ & $a_{4}$ \\
\hline 1 & 88 & 61 & 58 & 62 & 23 & 74 & 42 & 46 & 25 & 27 & 20 & 24 & 33 & 31 & 9 & 24 \\
\hline 2 & 84 & 63 & 39 & 62 & 75 & 39 & 33 & 49 & 37 & 49 & 20 & 35 & 36 & 47 & 23 & 35 \\
\hline 3 & 91 & 79 & 55 & 75 & 50 & 47 & 23 & 40 & -3 & -93 & -7 & -34 & -4 & -83 & -23 & -37 \\
\hline Av. & 88 & 64 & 51 & 66 & 49 & 53 & 33 & 45 & 43 & 38 & 20 & 29 & 34 & 39 & 16 & 29 \\
\hline
\end{tabular}

Overall, for the (A)-(CL) case, the maximum reductions show larger percentages than the ones achieved in (A)-(OL), with the maximal average ET reduction being measured on the index finger at a $75 \%$, while ET frequency was reduced at a maximal $70 \%$ on the forearm, with the respective reduction on the index remaining at the lower level of $42 \%$.

\subsection{Prototype (B) Open-Loop Evaluation}

The open-loop evaluation of iteration (B) and the effect of applying resisting force on the metacarpal region was performed via an experiment examining the ET suppression for variable increases in pressure while the volunteer's hand was in a straight position. The results, as defined in the previous subsections, are presented in Figure 15.

Initially and before the increase in pressure, a similar tremor pattern is observed mainly on the index finger, reaching high-amplitude ET close to $5 \mathrm{~Hz}$, while the amplitude is lower on the thumb and even lower on forearm points but under the same approximate frequency. Similar to the first (A)-(OL) experiment, the pressure follows an increasing and decreasing manner, reaching a maximum of approx. 6 bar, while the force alternates between 0-6 N. During the time period where the force is slowly increased (5-22 s), an increase in ET amplitude and frequency is observed at all four monitored parts of the hand. Interestingly, for the interval 11-14 s and during the stepwise increase in pressure, a sudden increase in ET is more evident. Then, closing with force stabilization, the ET amplitude and frequency gradually decrease, reaching a minimum during 27-33 s, when they gradually increase again on all four points with the sudden drop in pressure. 

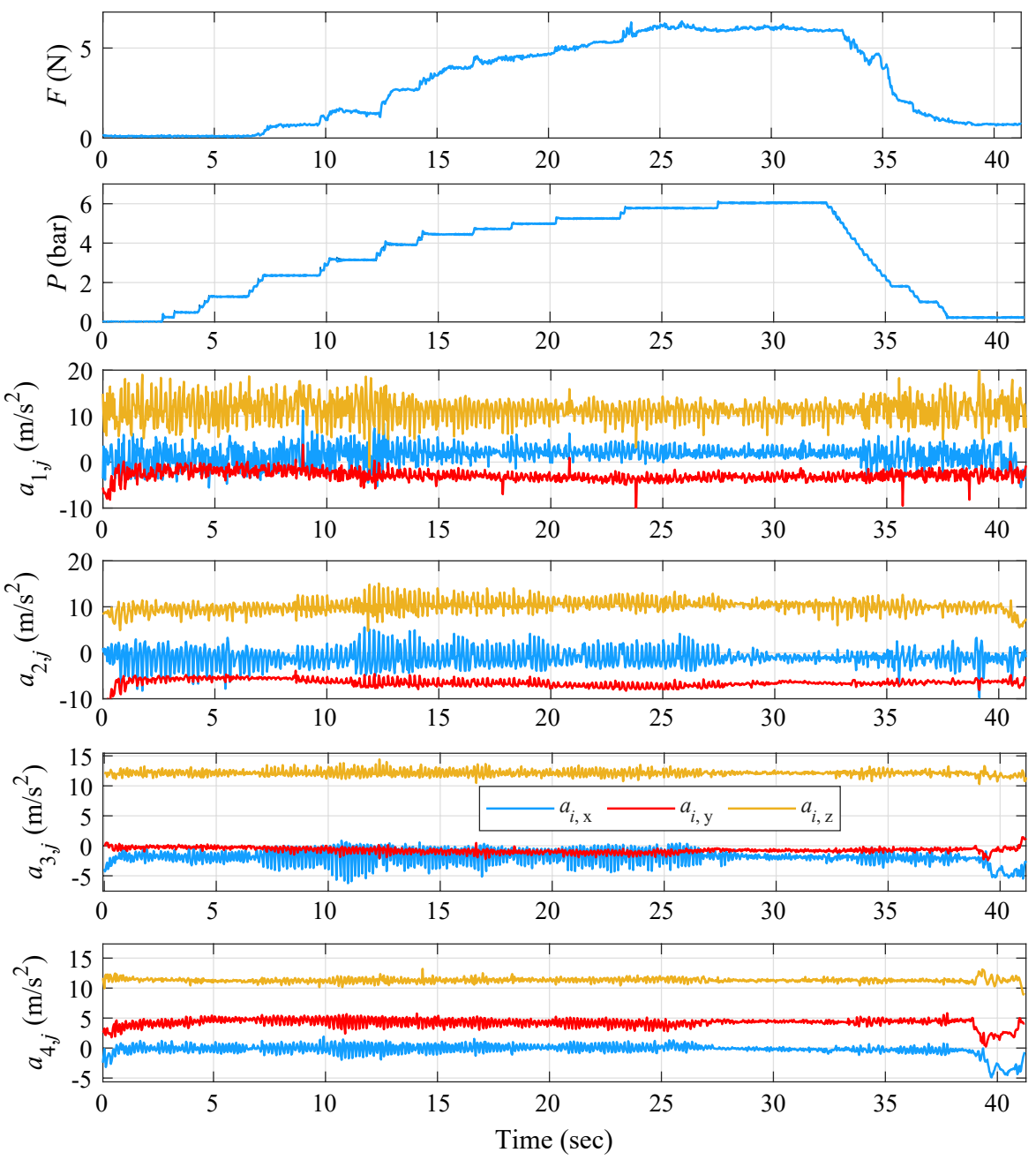

Figure 15. Experimental evaluation of iteration (B) and the effect of applying resisting force on the ET-diagnosed volunteer's metacarpal under open-loop reference in applied pressure and while the hand is at extended pose; Exp. 1.

As shown in Table 8 for the (B)-(OL) case, the application of resistive force on the metacarpal region still manages to effectively reduce ET frequency, but on lower maximum percentages than the ones achieved via (A). Specifically, the maximum reduction rates are encountered on the upper forearm, with $50 \%$ frequency. Reductions occur on all four measurement points, although frequency reduction on the index and thumb stays under $30 \%$. The largest tremor frequency reduction is observed on the $\mathrm{z}$ axis of accelerometer placed on the upper forearm and amounts to $70 \%$.

Table 8. ET frequency reduction rates during the Prototype (B) Open-Loop evaluation.

\begin{tabular}{lllllllllllllllll}
\hline Exp. & $f_{1, x}$ & $f_{1, y}$ & $f_{1, z}$ & $f_{1}$ & $f_{2, x}$ & $f_{2, y}$ & $f_{2, z}$ & $f_{2}$ & $f_{3, x}$ & $f_{3, y}$ & $f_{3, z}$ & $f_{3}$ & $f_{4, x}$ & $f_{4, y}$ & $f_{4, z}$ & $f_{4}$ \\
\hline 1 & 28 & 15 & 35 & 26 & 35 & 22 & 28 & 28 & 23 & 46 & 56 & 42 & 40 & 40 & 70 & 50 \\
\hline
\end{tabular}

By observing Table 9, and unlike the ET frequency, ET amplitude is mainly suppressed on the fingers by $56 \%$ and $53 \%$, while for the lower part of the forearm, the reduction rate drops to $8 \%$. The upper forearm tremor amplitude is reduced almost proportionally to its frequency at a rate of $44 \%$. Although there are reduction percentages near $70 \%$ on all four measured hand parts, $a_{2, x}$ is reduced significantly by $92 \%$. 
Table 9. ET amplitude reduction rates during the Prototype (B) Open-Loop evaluation.

\begin{tabular}{|c|c|c|c|c|c|c|c|c|c|c|c|c|c|c|c|c|}
\hline Exp. & $a_{1, x}$ & $a_{1, y}$ & $a_{1, z}$ & $a_{1}$ & $a_{2, x}$ & $a_{2, y}$ & $a_{2, z}$ & $a_{2}$ & $a_{3, x}$ & $a_{3, y}$ & $a_{3, z}$ & $a_{3}$ & $a_{4, x}$ & $a_{4, y}$ & $a_{4, z}$ & $a_{4}$ \\
\hline 1 & 70 & 62 & 31 & 56 & 92 & 27 & 41 & 53 & 73 & 76 & 8 & 52 & 52 & 73 & 7 & 44 \\
\hline
\end{tabular}

\subsection{Prototype (B) Closed-Loop Evaluation}

Similar to the (A)-(CL) case, the closed-loop evaluation of iteration (B) was initiated with a set-point reference force value set at approximately $6 \mathrm{~N}$, as obtained from (B)-(OL) as a suitable force level for effective ET reduction, while the volunteer's hand was in the relaxed state. The gain scheduler's control parameters were defined in the same manner as described in Section 5.2. The aforementioned parameters are presented in Table 10.

Table 10. PID parameters for the Prototype (B) Closed-Loop control scenario.

\begin{tabular}{lllll}
\hline $\mathbf{m}$ & $\begin{array}{l}\text { Operating } \\
\text { Regions [N] }\end{array}$ & $\boldsymbol{K}_{P, m}$ & $\boldsymbol{K}_{I, m}$ & $\boldsymbol{K}_{\boldsymbol{D}, \boldsymbol{m}}$ \\
\hline 1 & $F \in[0,2]$ & 1.20 & 0.10 & 0.02 \\
2 & $F \in(2,4]$ & 1.40 & 0.10 & 0.02 \\
3 & $F \in(4,6]$ & 1.65 & 0.22 & 0.07 \\
4 & $F \in(6,8]$ & 1.93 & 0.10 & 0.07 \\
5 & $F \in(8,10]$ & 1.70 & 0.20 & 0.05 \\
\hline
\end{tabular}

From the results presented in Figure 16, the initially high ET significantly decreased for all measurement points once the force approached the reference signal. The same behavior continues until the controller's deactivation at $44.5 \mathrm{~s}$, for all points except the index finger, where a small increase in ET amplitude is observed at $15 \mathrm{~s}$, when the volunteer performed an involuntary finger movement, Finally, ET properties rise again to the same levels when disabling the controller.

An additional experiment was performed while in closed-loop operation, where the controller was again activated in the presence of ET and the volunteer was asked to bend his fingers until they touched a spherical object and then to return them to their original position. This scenario was repeated two times. For the total duration of the experiment and throughout the volunteer's motion, the control was fine-tuned to provide smooth and fast behavior with the absence of abrupt alterations in pressure. From the results presented in Figure 17, it is clearly observed that during both controller activation periods 6-29 s and 40-66 s, ET amplitude decreases at all measurement points. The change of ET in the upper forearm approximates the corresponding diagrams of the lower forearm, although its frequency shows a larger decreasing tendency than that of the lower forearm. Interestingly, and in contrast to Figure 13 observations, the forearm's ET does not occur during the two control operations from 30 to $40 \mathrm{~s}$.

By observing Table 11, the largest mean decrease of tremor on the index finger appears on $y$ axis with $46 \%$. The highest decrease on the $\mathrm{x}$ and $\mathrm{y}$ axes appear in experiment 2 with percentages of $38 \%$ and $51 \%$, respectively, while on the $z$ axis, it is in experiment 1 with $48 \%$. Both the experiments managed to reduce index ET frequency by $41 \%$. Significant frequency tremor suppression is achieved on the thumb by $64 \%$ on the $y$ and $\mathrm{z}$ axes, and with a $58 \%$ reduction in experiment 1 . On the lower forearm, the maximum reduction is found on the $\mathrm{z}$ axis and at a rate of $75 \%$, while the reduction rates on the $\mathrm{x}$ and $\mathrm{y}$ axes are at $35 \%$ and $53 \%$, respectively. The largest decrease is observed in experiment 1 and on the $\mathrm{z}$ axis with $77 \%$. Experiment 1 has the optimum rate of reduction of the frequency of tremor with $67 \%$. On the upper forearm, as on the lower, the maximum reduction is detected on the $\mathrm{z}$ axis with $67 \%$. The highest reduction on the $\mathrm{x}$ and axes is observed in experiment 1 with 78 and $70 \%$, while on $\mathrm{z}$ axis, it is in experiment 2 at $76 \%$. Experiment 1 proves to be the most effective in the case of ET frequency suppression with $69 \%$. 

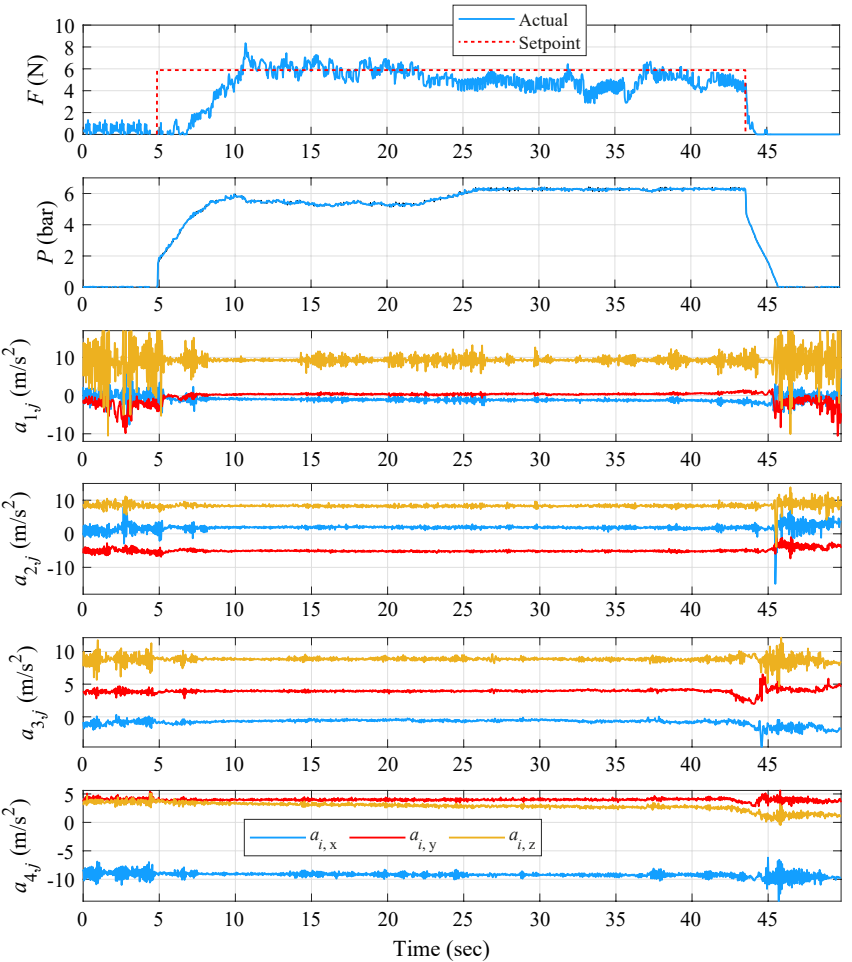

Figure 16. Experimental evaluation of iteration (B) and the effect of applying resisting force on the ET-diagnosed volunteer's metacarpal under closed-loop reference in applied pressure and while the hand is in a straight position; Exp. 1.
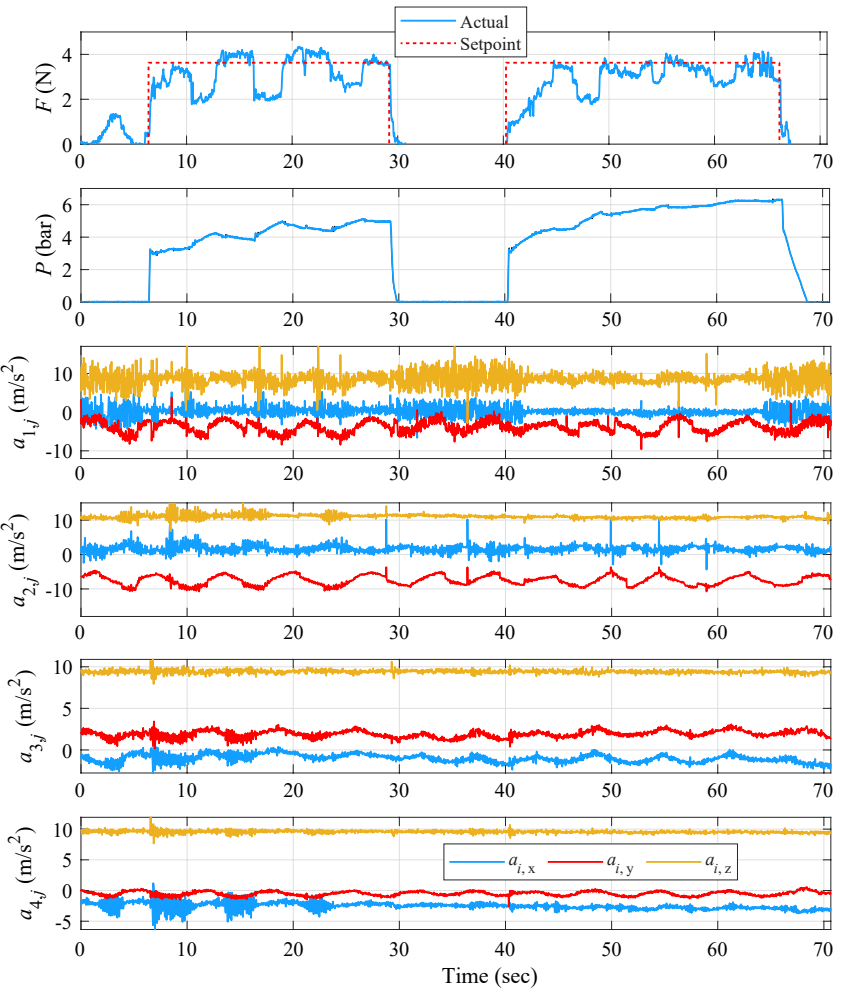

Figure 17. Experimental evaluation of iteration (B) and the effect of applying resisting force on the ET-diagnosed volunteer's metacarpal region under closed-loop reference in applied pressure, while performing finger-bending motions that finish with touching a spherical object; Exp. 2. 
Table 11. ET frequency reduction rates during the Prototype (B) Closed-Loop evaluation.

\begin{tabular}{lllllllllllllllll}
\hline Exp. & $f_{1, x}$ & $f_{1, y}$ & $f_{1, z}$ & $f_{1}$ & $f_{2, x}$ & $f_{2, y}$ & $f_{2, z}$ & $f_{2}$ & $f_{3, x}$ & $f_{3, y}$ & $f_{3, z}$ & $f_{3}$ & $f_{4, x}$ & $f_{4, y}$ & $f_{4, z}$ & $f_{4}$ \\
\hline 1 & 33 & 42 & 48 & 41 & 44 & 65 & 66 & 58 & 56 & 67 & 77 & 67 & 78 & 70 & 58 & 69 \\
2 & 38 & 51 & 35 & 41 & 24 & 64 & 62 & 50 & 14 & 40 & 73 & 42 & 22 & 41 & 76 & 46 \\
\hline Av. & 35 & 46 & 41 & 41 & 34 & 64 & 64 & 54 & 35 & 53 & 75 & 54 & 50 & 55 & 67 & 57 \\
\hline
\end{tabular}

According to Table 12, the largest decrease in tremor amplitude noticed on the index is observed on average on the $x$ axis with $79 \%$. However, The largest decrease is observed on the $y$ axis and in experiment 1 with $91 \%$. In experiment 1 , tremor amplitude is reduced optimally with $73 \%$. As for the thumb, the largest reduction in tremor amplitude is observed on the $\mathrm{x}$ axis with $55 \%$, significantly higher than the other two axes. The largest decrease on the $\mathrm{x}$ axis is found in experiment 2 at $75 \%$. The ET amplitude is reduced by almost half during the first experiment. The maximum decrease on the lower forearm appears on the $\mathrm{x}$ axis with $48 \%$. The largest tremor amplitude decrease on the $\mathrm{x}$ axis is observed in experiment 1 with 50\%, while both experiments managed to reduce tremor amplitude by almost $30 \%$. On the upper forearm, the maximum reduction is again detected on the $\mathrm{x}$ axis at $36 \%$, while the reduction rates on the $\mathrm{z}$ axis remains close with $35 \%$. The largest decrease is observed in experiment 2 on the $x$ axis with $59 \%$. Experiment 2 appears to have the highest reduction rate at $38 \%$.

Table 12. ET amplitude reduction rates during the Prototype (B) Closed-Loop evaluation.

\begin{tabular}{|c|c|c|c|c|c|c|c|c|c|c|c|c|c|c|c|c|}
\hline Exp. & $a_{1, x}$ & $a_{1, y}$ & $a_{1, z}$ & $a_{1}$ & $a_{2, x}$ & $a_{2, y}$ & $a_{2, z}$ & $a_{2}$ & $a_{3, x}$ & $a_{3, y}$ & $a_{3, z}$ & $a_{3}$ & $a_{4, x}$ & $a_{4, y}$ & $a_{4, z}$ & $a_{4}$ \\
\hline 1 & 76 & 91 & 52 & 73 & 36 & 21 & 20 & 50 & 50 & 13 & 24 & 29 & 14 & 4 & 42 & 20 \\
\hline 2 & 83 & 22 & 41 & 49 & 75 & 9 & 18 & 34 & 47 & 32 & 12 & 30 & 59 & 27 & 29 & 38 \\
\hline Av. & 79 & 56 & 46 & 61 & 55 & 15 & 19 & 42 & 48 & 22 & 18 & 29 & 36 & 15 & 35 & 29 \\
\hline
\end{tabular}

\section{Conclusions}

In this article, a soft exoskeletal glove for investigating Essential Tremor (ET) suppression was proposed. The glove setup concept followed a patch-and-use logic by avoiding the use of complex mechanisms comprising hard and heavy materials, while increasing the ease of installation and use without lowering the durability factor. For actuation, Pneumatic Artificial Muscles (PAMs) were utilized for their important advantages such as inherent compliance and high power-to-mass ratio. To investigate the glove's potential in reducing ET via application of resisting forces on different parts of a user's lower arm, two design iterations were implemented to apply localized force to the (A) index finger and (B) metacarpal region, while measuring the acceleration of four points including the distal phalanges of the index and thumb along with metacarpal and forearm regions. To this goal, a data acquisition setup was implemented and utilized for acquiring acceleration measurements in order to determine the effectiveness of the suppression iterations.

Both iterations were extensively evaluated under various motion scenarios by a chronic ET-diagnosed volunteer. The performed experimental sequences involved the prototypes' evaluation initially under open-loop control of the kinesthetic force, and subsequently under closed loop sequences incorporating a PID control scheme. Specifically, regarding the tremor suppression attempts, iteration (A) showed a visible decrease in ET frequency and amplitude in the cases where the force reached a specific plateau, reaching a maximal decrease in metacarpal ET frequency and index finger ET amplitude of 70\% and $75 \%$, respectively. Using these plateau values as reference forces, closed-loop sequences incorporating a PID control scheme were performed, which further proved the setup methodology's efficiency in suppressing ET, even in cases where the reference force was a constant setpoint and when the volunteer was performing voluntary movements with object-touching actions. Reductions in ET were recorded in all investigated cases, which 
reached a maximal reduction rate of 75 and $70 \%$ for the index ET amplitude and forearm ET frequency, respectively. Similarly, and in accordance with the open-loop case, the index finger appeared to have the largest tremor amplitude reduction, thus proving the effect of the experimental strategy. At the same time, the maximum ET frequency reduction was observed in the forearm region, a part of the hand where, for iteration (A), the force is not directly exercised on.

Regarding the open-loop evaluation of iteration (B) and the application of force directly on the metacarpal area, the experiment conducted resulted in approximately $50 \%$ reduction in ET amplitude for all four measured parts of the hand. Furthermore, the device was capable of halving the metacarpal's and forearm's tremor frequency, while lower reduction rates were achieved for the two fingers. During the closed-loop evaluation for iteration (B), ET frequency reduction rates followed a similar pattern to the open-loop case scenario. The ET frequency of metacarpal and forearm areas was reduced by almost $70 \%$. In parallel, the index and thumb fingers' frequencies were also decreased but with the reduction rates not exceeding $58 \%$. Finally, ET amplitude was reduced by $73 \%$ on the index finger and by $50 \%$ on the thumb, while the respective percentages for the metacarpal and forearm regions stayed below $40 \%$.

The investigation showed that both PAM-enabled approaches, regarding kinesthetic force exertion on the metacarpal and index finger, were able to effectively reduce ET via constant setpoint forces, with the metacarpal solution providing slightly less reductions during closed-loop control but with the important advantage of not constricting finger movements.

Moreover, during the experimental trials and results, an interesting finding was made with the increase in ET amplitude in the metacarpal and forearm areas simultaneously with the tremor reduction in the fingers. This "tremor translation" phenomenon was observed only for iteration (A), which seeks to repress the tremor by exerting force directly to the fingers, and not for iteration (B), where the force directly affects the metacarpal region.

All presented findings highlight the exoskeletal glove's potential for future investigations involving different tremor types. Further experimentation on male/female volunteers of different ages, clinical trials on volunteers suffering from various tremor-causing neurological disorders (e.g., Parkinson's), validation of safety during alternative and more intense active motion strategies, as well as implementation of more advanced model-based algorithms for active force and compliance control are considered a necessity for further and future evaluation of the proposed approach.

Author Contributions: Conceptualization, G.A. and V.S.; methodology, V.S. and G.A.; software, V.S.; validation, V.S.; formal analysis, V.S. and G.A.; investigation, V.S.; resources, G.A. and S.M.; data curation, V.S.; writing—original draft preparation, V.S.; writing—review and editing, G.A. and S.M.; visualization, G.A.; supervision, G.A. and S.M. All authors have read and agreed to the published version of the manuscript.

Funding: This research received no external funding.

Institutional Review Board Statement: The study was conducted according to the guidelines of the Declaration of Helsinki, and approved by the Institutional Review Board of University of Patras (protocol code 13976, 3 October 2019).

Informed Consent Statement: Informed consent was obtained from all subjects involved in the study.

Conflicts of Interest: The authors declare no conflict of interest.

\section{Abbreviations}

The following abbreviations are used in this manuscript:

ET Essential tremor

DBS Deep Brain Stimulation

PAM Pneumatic Artificial Muscle 
PID Proportional-Integral-Derivative

SPI Serial Peripheral Interface

SISO Single-Input Single-Output

\section{References}

1. McAuley, J.H. Physiological and pathological tremors and rhythmic central motor control. Brain 2000, 123, 1545-1567. [CrossRef] [PubMed]

2. Agarwal, S.; Biagioni, M.C. Essential Tremor; StatPearls Publishing: Treasure Island, FL, USA, 2021.

3. Louis, E.D.; Ottman, R. How Many People in the USA Have Essential Tremor? Deriving a Population Estimate Based on Epidemiological Data. Tremor Other Hyperkinetic Movements 2014, 4, 259. [CrossRef] [PubMed]

4. Elble, R.J. What is essential tremor? Topical collection on movement disorders. Curr. Neurol. Neurosci. Rep. 2013, 13, 1-8. [CrossRef] [PubMed]

5. Zesiewicz, T. Overview of essential tremor. Neuropsychiatr. Dis. Treat. 2010, 6, 401. [CrossRef] [PubMed]

6. Zhou, Y.; Jenkins, M.E.; Naish, M.D.; Trejos, A.L. Development of a Wearable Tremor Suppression Glove. In Proceedings of the 2018 7th IEEE International Conference on Biomedical Robotics and Biomechatronics (Biorob), Enschede, The Netherlands, 26-29 August 2018; pp. 640-645. [CrossRef]

7. Turkistani, A. Development of an Effective Portable and Flexible Glove for Hand Tremor Suppression. Master's Thesis, Western Michigan University, Kalamazoo, MI, USA, 2017.

8. Awantha, W.V.; Wanasinghe, A.T.; Kavindya, A.G.; Kulasekera, A.L.; Chathuranga, D.S. A Novel Soft Glove for Hand Tremor Suppression: Evaluation of Layer Jamming Actuator Placement. In Proceedings of the 2020 3rd IEEE International Conference on Soft Robotics (RoboSoft), New Haven, CT, USA, 15 May-15 July 2020; pp. 440-445. [CrossRef]

9. Loureiro, R.C.; Belda-Lois, J.M.; Lima, E.R.; Pons, J.L.; Sanchez-Lacuesta, J.J.; Harwin, W.S. Upper Limb Tremor Suppression in ADL Via an Orthosis Incorporating a Controllable Double Viscous Beam Actuator. In Proceedings of the 9th International Conference on Rehabilitation Robotics, Chicago, IL, USA, 28 June-1 July 2005; pp. 119-122. [CrossRef]

10. Yi, A.; Zahedi, A.; Wang, Y.; Tan, U.X.; Zhang, D. A novel exoskeleton system based on magnetorheological fluid for tremor suppression of wrist joints. IEEE Comput. Soc. 2019, 2019, 1115-1120. [CrossRef]

11. Hosseini, S.; Al-Jumaily, A. Active Force Control System for Tremor Suppression in Elbow Joint. In Proceedings of the 2016 IEEE International Symposium on Robotics and Intelligent Sensors (IRIS), Tokyo, Japan, 17-20 December 2016; pp. 140-145. [CrossRef]

12. Manto, M.; Topping, M.; Soede, M.; Sanchez-Lacuesta, J.; Harwin, W.; Pons, J.; Williams, J.; Skaarup, S.; Normie, L. Dynamically Responsive Intervention for Tremor Suppression. IEEE Eng. Med. Biol. Mag. 2003, 22, 120-132. [CrossRef] [PubMed]

13. Gallego, J.A.; Rocon, E.; Ibáñez, J.; Dideriksen, J.L.; Koutsou, A.D.; Paradiso, R.; Popovic, M.B.; Belda-Lois, J.M.; Gianfelici, F.; Farina, D.; et al. A soft wearable robot for tremor assessment and suppression. In Proceedings of the 2011 IEEE International Conference on Robotics and Automation, Shanghai, China, 9-13 May 2011; pp. 2249-2254. [CrossRef]

14. Rocon, E.; Ruiz, A.F.; Pons, J.L.; Belda-Lois, J.M.; Sánchez-Lacuesta, J.J. Rehabilitation robotics: A wearable exo-skeleton for tremor assessment and suppression. In Proceedings of the 2005 IEEE International Conference on Robotics and Automation, Barcelona, Spain, 18-22 April 2005; Volume 2005, pp. 2271-2276. [CrossRef]

15. Liu, W.; Kai, T.; Kiguchi, K. Tremor Suppression with Mechanical Vibration Stimulation. IEEE Access 2020, 8, $226199-226212$. [CrossRef]

16. Huen, D.; Liu, J.; Lo, B. An Integrated Wearable Robot for Tremor Suppression with Context Aware Sensing. In Proceedings of the 2016 IEEE 13th International Conference on Wearable and Implantable Body Sensor Networks (BSN), San Francisco, CA, USA, 14-17 June 2016; pp. 312-317. [CrossRef]

17. Rocon, E.; Belda-Lois, J.M.; Ruiz, A.F.; Manto, M.; Moreno, J.C.; Pons, J.L. Design and validation of a rehabilitation robotic exoskeleton for tremor assessment and suppression. IEEE Trans. Neural Syst. Rehabil. Eng. 2007, 15, 367-378. [CrossRef] [PubMed]

18. Kiguchi, K.; Hayashi, Y. Upper-limb tremor suppression with a 7DOF exoskeleton power-assist robot. In Proceedings of the 2013 35th Annual International Conference of the IEEE Engineering in Medicine and Biology Society (EMBC), Osaka, Japan, 3-7 July 2013; Volume 2013, pp. 6679-6682. [CrossRef]

19. Ohara, E.; Yano, K.; Horihata, S.; Aoki, T.; Nishimoto, Y. Development of Tremor-Suppression filter for Meal-Assist robot. In Proceedings of the World Haptics 2009-Third Joint EuroHaptics Conference and Symposium on Haptic Interfaces for Virtual Environment and Teleoperator Systems, Salt Lake City, UT, USA, 18-20 March 2009; pp. 238-243. [CrossRef]

20. Emma Watch: A Device to Ease Tremors, Project Emma, Microsoft. 2017. Available online: https://www.microsoft.com/en-us/ research/project/project-emma/ (accessed on 24 August 2021).

21. Gyenno Spoon. Official Australian Gyenno Spoon Distributor, Gyenno Australia \& New Zealand. 2016. Available online: https: / / www.gyenno.com.au/ (accessed on 24 August 2021).

22. Yusop, Z.M.; Zain, M.Z.M.; Hussein, M.; As'Arry, A. Performance of a passive device in improving tremor patients' handwriting capability. In Proceedings of the 2012 International Conference on Biomedical Engineering (ICoBE), Penang, Malaysia, 27-28 February 2012; pp. 400-403. [CrossRef]

23. Zhou, Y.; Ibrahim, A.; Jenkins, M.; Naish, M.D.; Trejos, A.L. Analysis of the Effect of Common Disturbances on the Safety of a Wearable Tremor Suppression Device. IEEE Robot. Autom. Lett. 2021, 26, 2846-2853. [CrossRef] 
24. Andrikopoulos, G.; Arvanitakis, J.; Manesis, S.; Nikolakopoulos, G. A switched system modeling approach for a Pneumatic Muscle Actuator. In Proceedings of the 2012 IEEE International Conference on Industrial Technology, Athens, Greece, 19-21 March 2012; pp. 833-839. [CrossRef]

25. Skaramagkas, V.; Andrikopoulos, G.; Manesis, S. An Experimental Investigation of Essential Hand Tremor Suppression via a Soft Exoskeletal Glove. In Proceedings of the 2020 European Control Conference (ECC), St. Petersburg, Russia, 12-15 May 2020; pp. 889-894. [CrossRef]

26. Choi, H.S.; Lee, C.H.; Baek, Y.S. Design of a pneumatic actuated ankle-foot orthosis which has talocrural and subtalar joint. IEEE Comput. Soc. 2019, 2019, 276-281. [CrossRef]

27. Lei, J.; Zhu, J. Pneumatic Artificial Muscles Force Modelling and the Position and Stiffness Control on the Knee Joint of the Musculoskeletal Leg. Int. J. Bioautom. 2017, 21, 31-42.

28. Andrikopoulos, G.; Nikolakopoulos, G.; Manesis, S. An Experimental Study on Thermodynamic Properties of Pneumatic Artificial Muscles. In Proceedings of the 20th Mediterranean Conference on Control and Automation, Barcelona, Spain, 3-6 July 2012; pp. 1334-1340. [CrossRef]

29. Fuller, A.T. A History of Control Engineering 1800-1930. Electron. Power 1979, 25, 651. [CrossRef]

30. Andrikopoulos, G.; Nikolakopoulos, G.; Manesis, S. Non-linear control of pneumatic artificial muscles. In Proceedings of the 21st Mediterranean Conference on Control and Automation, Platanias, Greece, 25-28 June 2013; pp. 729-734. [CrossRef] 